\title{
Levels of p53 expression determine the competitive ability of embryonic stem cells during the onset of differentiation.
}

Salvador Perez Montero", \#, Sarah Bowling1, 2, Rubén Pérez-Carrasco 2, \#,* and Tristan A. Rodriguez ${ }^{1, *}$.

${ }^{1}$ National Heart and Lung Institute, Imperial College London, UK

${ }^{2}$ Current address: Stem Cell Program, Boston Children's Hospital, Boston, MA, USA and Department of Stem Cell and Regenerative Biology, Harvard University, Cambridge, MA, USA

${ }^{3}$ Department of Life Sciences, Imperial College London, UK

\# Both these authors contributed equally.

*Authors for correspondence:

r.perez-carrasco@imperial.ac.uk and tristan.rodriguez@imperial.ac.uk 


\section{ABSTRACT}

During development, the rate of tissue growth is determined by the relative balance of cell division and cell death. Cell competition is a fitness quality control mechanism that contributes to this balance by eliminating viable cells that are less-fit than their neighbours. What mutations confer cells with a competitive advantage or the dynamics of the interactions between winner and loser cells are not well understood. Here, we show that embryonic cells lacking the tumour suppressor $p 53$ are super-competitors that eliminate their wild-type neighbours through the direct induction of apoptosis. This elimination is context dependant, as does not occur when cells are pluripotent and is triggered by the onset of differentiation. Furthermore, by combining mathematical modelling and cell-based assays we show that the elimination of wild-type cells is not through a competition for space or nutrients, but instead is mediated by short range interactions that are dependent on the local cell neighbourhood. This highlights the importance of the local cell neighbourhood and the competitive interactions within this neighbourhood for the regulation of proliferation during early embryonic development.

Running title: Cell competition and P53

Keywords: cell competition, p53, mathematical modelling. 


\section{INTRODUCTION}

2 The regulation of growth and tissue homeostasis during embryogenesis relies on the proper

3 balance of cell division and cell death. One mechanism contributing to this balance is cell

4 competition. Cell competition is a quality control mechanism conserved from Drosophila to

5 mammals that eliminates cells that although viable, are less fit than their neighbours. During

6 competition those cells that are eliminated are generically termed losers. Accompanying this

7 elimination, the fitter cells (winners) undergo compensatory proliferation, maintaining tissue

8 homeostasis -reviewed in (Baker, 2020; Bowling et al., 2019; Diaz-Diaz and Torres, 2019;

9 Johnston, 2014; Madan et al., 2018; Maruyama and Fujita, 2017; Vishwakarma and Piddini,

10 2020). For the purpose of this study, we define cell fitness as a cell's ability to thrive in its

11 environment. This ability is likely to be determined by a number of parameters, including

12 signalling ability, metabolic rates and cell adhesion properties, but ultimately is established

13 by the balance between the cell division rate and the sensitivity to cell death.

14 An important implication of cell competition is that cellular fitness is not only a cell-intrinsic

15 property but is also determined relative to the fitness of neighbouring cells- a cell that is of

16 sub-optimal fitness in one context may be 'super-fit' in the context of a different cell

17 population. This is most clearly demonstrated in the case of wild-type cells, that in

18 Drosophila and in mouse can eliminate a range of defective cells -reviewed in (Bowling et

19 al., 2019), but can in turn also be eliminated by cells that over-express Myc (Claveria et al.,

20 2013; de la Cova et al., 2004; Moreno and Basler, 2004; Sancho et al., 2013; Villa Del

21 Campo et al., 2014). Due to this ability to induce the elimination of wild-type cells, cells over-

22 expressing $M y c$ have been termed super-competitors. The observation that cell fitness is

23 relative to the cells neighbours implies that cells can interpret their relative fitness levels

24 during cell competition. However, little is known regarding the mechanisms by which this

25 process takes place. In Drosophila and cancer cells differential expression of Flower

26 isoforms can act as fitness fingerprints for the cells winner and loser status (Madan et al.,

27 2019; Rhiner et al., 2010). Similarly, in Drosophila innate immune-like signalling has been

28 shown to induce for the elimination of Minute cells (that have a ribosomal deficiency) when

29 they are surrounded by wild-type cells and for the elimination of wild-type cells by Myc

30 super-competitors (Meyer et al., 2014). However, this pathway is not required for

31 competition when the flies are maintained in a sterile environment (Germani et al., 2018) and

32 promotes the overgrowth of polarity deficient cells when they are surrounded by wild-type

33 cells (Katsukawa et al., 2018).

34 In mouse, at the onset of embryonic differentiation cell competition has been demonstrated

35 to mediate the elimination of defective cells (Sancho et al., 2013) as well as those cells with

36 low levels of Myc expression (Claveria et al., 2013). In the post-implantation embryo, just 
37 prior to gastrulation, this process eliminates around $35 \%$ of embryonic cells due to

38 repression of the mTOR pathway (Bowling et al., 2018). This large-scale elimination is

39 thought to ensure that only the fittest cells go on to contribute to further development and the

40 germline (Bowling et al., 2019), and one important trigger of this elimination is mitochondrial

41 dysfunction and mitochondrial DNA mutations (Lima et al., 2021). In the pre-implantation

42 mouse embryo signalling via the Hippo pathway is required for the elimination through cell

43 competition of mis-patterned cells (Hashimoto and Sasaki, 2019) and this pathway also

44 mediates the elimination of normal human embryonic stem cells (ESCs) by karyotypically

45 abnormal ones (Price et al., 2021), explaining how the abnormal cells expand in human

46 pluripotent stem cell cultures.

47 Importantly, the cells eliminated in the early post-implantation mouse embryo display 48 elevated P53 levels (Bowling et al., 2018; Lima et al., 2021) and cells with increased P53 49 expression are also eliminated during mouse during organogenesis (Zhang et al., 2017). In 50 Madin-Darby Canine Kidney (MDCK) cells, mutation of the cell polarity gene Scribble in a 51 mosaic fashion leads to an increase in P53 that induces mutant cell elimination via 52 mechanical cell competition (Wagstaff et al., 2016). The observation that in mouse chimeras, 53 p53 mutant cells have a selective advantage during development (Dejosez et al., 2013), 54 suggests that loss of P53 expression provides cells with a competitive advantage. A further 55 fascinating indication of the role of P53 in competition comes from analysing interspecies 56 chimeras as well as co-cultured human and mouse pluripotent stem cells. Both in vivo and in 57 vitro, human ESCs are eliminated by mouse ESCs by cell competition. However, mutation of 58 p53 in the human cells is sufficient to prevent the human ESC elimination (Zheng et al., 59 2021). Together, these data suggest that relative P53 levels are a key determinant of 60 embryonic fitness. Here we have asked if in mouse, mutation of p53 turns cells into super61 competitors. By comparing the behaviour of wild-type and p53 mutant cells in separate 62 versus coculture conditions and by developing a mathematical model of this interaction, we 63 demonstrate that $p 53$ null mutant mouse ESCs actively induce the apoptotic elimination of 64 wild-type cells in co-culture. Furthermore, we also find that this elimination is mediated by 65 short-range signalling, highlighting the importance of local competitive interactions for the 66 regulation of cell proliferation during the onset of differentiation.

\section{RESULTS}

\section{P53 mutant ESCs behave as super-competitors}

69 The observation that in the embryo and in ESC models of cell competition loser cells show 70 an increase in P53 expression (Bowling et al., 2018; Lima et al., 2021) combined with the 71 finding that in chimeras p53 mutant cells contribute preferentially to the embryo (Dejosez et 
72 al., 2013) suggests that differences in the levels of P53 expression determine the

73 competitive ability of embryonic cells. To test this possibility, we generated $p 53$ null mutant

74 mouse ESCs (Fig. 1A) and compared their behaviour when they were cultured in a

75 homogeneous (separate) culture to when they were co-cultured with wild-type cells. We

76 found that in pluripotency culture conditions, p53 mutant cells grew at a similar rate to cells

77 both in separate and co-culture conditions (Fig. 1B-C). Here $8 \times 10^{5}$ cells of each genotype

78 were plated in separate culture and the co-culture contained a mix of $4 \times 10^{5}$ cells of each

79 genotype, so also totalling $8 \times 10^{5}$ cells. However, in contrast to this, when the same cell

80 numbers were plated and the ESCs were induced to differentiate by culture in N2B27, $p 53^{-/}$

81 cells displayed a small proliferative advantage in separate culture and induced the growth

82 arrest of wild-type cells in co-culture (Fig. D-E). This behaviour was not due to the inability of

83 p53 mutant cells to initiate differentiation, as when cultured in N2B27 p53 ${ }^{-/}$cells down-

84 regulate the expression of naïve pluripotency markers and increase the expression of the 85 post-implantation epiblast marker Fgf5 in a similar way as control cells do (Fig. 1F). These

86 results suggest that mutation of p53 makes cells into super-competitors that out-compete 87 wild-type cells.

88 The cyclin dependant kinase inhibitor Cdkn1a (p21) is an important target activated by P53 89 (Pilley et al., 2021). To test if P21 regulation of the cell cycle is an important part of the 90 mechanism by which P53 confers a super-competitor status we generated p21 mutant ESCs 91 (Fig. S1A). We found that when these cells were cultured in N2B27 they could differentiate 92 normally (Fig. S1B) and grew similarly to control cells in both separate and co-culture 93 conditions (Fig. S1C-D), and therefore do not show super-competitor behaviour. The 94 observation that both $p 53^{-/}$and $p 21^{-/}$cells displayed similar levels of Edu incorporation to 95 control cells in both separate and co-culture with control cells (Fig. 1G and Fig. S1E) further 96 supports the conclusion that regulation of the cell cycle is not the primary mechanism by 97 which p53 mutant cells become super-competitors.

98 Given that we did not find evidence for differences in the cycle explaining the increased 99 competitive ability of $p 53$ mutant cells we investigated the role of the apoptotic response.

100 When we analysed cleaved-caspase 3 expression we observed that it was significantly 101 lower in $p 53^{-/}$ESCs when compared to control cells after 3 days and after 4 days culture in 102 N2B27 (Fig. 1H). This indicates that p53 mutant cells are intrinsically more resistant to 103 apoptosis than wild-type cells and likely explains their faster growth rate in separate culture. 104 Interestingly, we also observed that wild-type cells at day 4 of co-culture with $p 53^{-/}$ESCs 105 showed an increase in cleaved-caspase 3 expression compared to when they were 106 maintained in a homotypic (separate) culture (Fig. 1H). The fact that this difference was not 107 apparent at day 3 of co-culture, even though at this time-point there is a clear difference in 
108 the growth rate of $p 53^{-/}$and control ESCs, suggests that there may be caspase 3

109 independent death occurring before this stage. To address this possibility, we analysed the

110 expression of cleaved-caspase 8, that mediates extrinsic cell death signalling (Fuchs and

111 Steller, 2015). We not only found that $p 53^{-/}$cells displayed lower levels of cleaved caspase-

1128 expression than wild-type cells in separate culture (Fig. S2A-B), but also that at day 3 wild-

113 type cells showed higher cleaved-caspase 8 expression in co-culture compared to separate 114 culture (Fig. $1 \mathrm{I}$ and Fig. S2C). These results suggest that $p 53^{-/}$cells out-compete wild-type 115 cells by inducing their apoptotic elimination.

\section{Developing a mathematical model of super-competition}

117 Our results indicate that $p 53$ mutant cells outcompete wild-type cells in coculture. To gain 118 further insight into this competition and explore the mechanism by which loser cell 119 elimination takes place, we developed a mathematical model to recapitulate quantitatively 120 the differential cell population dynamics in separate and co-culture assays. The aim of this 121 model is twofold: on one hand disentangle potential confusing effects of population intrinsic 122 growth and cell-cell competition effects on total cell population growth, while at the same 123 time obtain mechanistic information of the nature of competition. We described the evolution 124 in time of the number of wild-type cells $(W)$ and $p 53^{\%}$ cells $(P)$ by specifying a set of ordinary 125 differential equations (ODE) describing the effect that different population compositions ( $W$, $126 \mathrm{P}$ ) have on the net growth of each species. In the absence of competition - at low cell 127 numbers - each species population grows with an intrinsic rate $\rho_{i}>0, i=\{W, P\}$ (Fig. 2A 128 top), this is the net growth of the population incorporating the intrinsic proliferation and 129 apoptotic rates. As the population grows, cells compete with each other with a strength $k_{j}$ 130 specific for each of the 4 possible interactions $(j=\{W W, W P, P W, P P\}$, see Fig $3 \mathrm{~A}-\mathrm{B})$,

$$
\begin{aligned}
& \frac{\mathrm{d} W}{\mathrm{~d} t}=\rho_{W} W\left(1-k_{W W} W-k_{W P} P\right) \\
& \frac{\mathrm{d} P}{\mathrm{~d} t}=\rho_{P} P\left(1-k_{P W} W-k_{P P} P\right)
\end{aligned}
$$

131 This Lotka-Volterra description has been successfully used in a different context of cell 132 competition (Ram et al., 2019) and assumes that the magnitude of the competition is 133 proportional to the number of cells of each type in the dish (or equivalently, their 134 concentration). Therefore, the increase in apoptotic rate in a crowded dish is translated into 135 a decrease of the total net growth rate of the population of each species, making it negative 136 in crowded environments (e.g. $\frac{d W}{d t}<0$, see Fig. 2A). To test the model, we used Bayesian 137 inference to identify which portion of the parameter space $\left\{\rho_{i}, k_{j}\right\}$ is consistent with the 138 experimental data by using a likelihood function that compares experimental and numerical 
139 trajectories (see Materials and Methods)(Toni et al., 2009). The result of this analysis is a 140 preliminary credibility distribution of the parameters of the model compatible with the cell 141 population trajectories shown in Fig. 1. Inspection of these distribution of parameters allowed 142 us to identify which additional experimental initial plating conditions would carry information 143 to improve our predicted parameter distributions. Experiments were then performed using 144 these identified plating conditions and were analysed in the same manner as described 145 above. This resulted in an iterative analysis composed of a set of experimental trajectories 146 for 24 different plating conditions that generated a posterior credibility distribution of the 147 parameters that can be used to extract mechanistic information of the competition (Fig. S3). 148 The trajectories corresponding to this distribution show a very good fit to the model (Fig. 2C 149 and Fig. S4). Analysis of the pairwise relationship between parameters confirm that the 150 intrinsic net growth of $p 53^{-/}$is faster than the intrinsic net growth of wild-type cells, $\rho_{P}>\rho_{W}$ 151 (Fig. 2D). In addition, comparison of the competition strengths revealed that there were not 152 significant differences between the competition of $p 53^{-/}$cells with themselves compared to 153 the corresponding homotypic competition between wild-type cells $\left(k_{W W} \simeq k_{P P}\right)$. On the other 154 hand, as expected, the presence of $p 53^{-}$ESCs induced a dramatic decrease on the growth 155 rate of wild-type cells $\left(k_{W W}<k_{W P}\right)$. Strikingly, $p 53^{-/}$ESC growth was unaffected by the 156 identity of the cells in their neighbourhood $\left(k_{P P} \simeq k_{P W}\right)$. Our model therefore quantified the 157 interactions between cells allowing us to identify differential cellular properties and compare 158 their magnitude.

\section{P53 mutant cells induce the apoptotic elimination of wild-type ESCs}

160 Our model indicates that $p 53^{-/}$ESCs have a direct negative effect on wild-type cells and the 161 increased expression of apoptotic markers in cocultured wild-type cells (Fig. 1H-I and S2A-

162 C) suggests that this takes place through the induction of their apoptotic elimination. To test 163 this possibility, we first performed the cell competition assays in the presence of a pan164 caspase inhibitor (Z-VAD-FMK). For this we cultured wild-type and $p 53^{-/}$ESCs in N2B27 in 165 separate and co-culture conditions and added the caspase inhibitor from day 2 to day 4 of 166 culture. We found that this partially rescued the elimination of wild-type cells in co-culture 167 (Fig. S2D), raising the prospect that non-apoptotic forms of cell death may be contributing to 168 the out-competition of wild-type cells.

169 BCL-2 is a key anti-apoptotic protein acting in the mitochondrial apoptotic pathway (Tait and 170 Green, 2013) and Bcl-2 over-expression prevents loser cell elimination in interspecies 171 chimeras (Zheng et al., 2021). We therefore generated doxycycline inducible ESCs (Fig. $1723 \mathrm{~A})$. Induction of BCL2 expression by doxycycline addition decreased the intrinsic apoptotic 173 rate to levels that were similar to those found in p53 mutant cells (Fig. 3B). In contrast to this 174 BCL2 induction did not significantly affect proliferation rates (Fig. 3C). We therefore assayed 
175 the behaviour of $B c /-2$ inducible ESCs $\left(B c / 2^{\text {Ind }}\right)$ in separate and co-culture with $p 53^{-/}$cells.

176 We found that similarly to what occurred with wild-type ESCs, when $B c / 2^{\text {Ind }}$ ESCs were

177 cultured separately in N2B27 without the addition of doxycycline they grew slightly slower

178 than $p 53^{-}$ESCs and when co-cultured with $p 53^{--}$ESCs they were effectively eliminated

179 (Fig. 3D-E). In contrast to this, we observed that upon BCL2 induction with doxycycline

$180 \mathrm{Bcl} \mathrm{I}^{\mathrm{Ind}}$ ESCs grew similarly to $\mathrm{p53^{-/ }}$ cells, both in separate and co-culture condition (Fig. 3F-

$181 \mathrm{G})$ and are therefore no longer eliminated by $p 53^{-/}$cells. These results indicate that $p 53$

182 mutant cells out-compete wild-type cells by inducing their apoptotic elimination.

183 To analyse the dynamics by which BCL2 rescues the competition, we used our model

184 together with the inferred parameters to reproduce experimental trajectories of doxycycline

185 induced rescue at different timepoints of the competition. The model successfully 186 reproduced the dynamics of the rescue (Fig. $3 \mathrm{H}$ ). Untreated $\mathrm{Bcl} 2^{\text {Ind }} \mathrm{ESC}$ were successfully

187 simulated using the same parameters as wild-type cells. In contrast to this, to reproduce the 188 experimental trajectories of $\mathrm{Bcl}^{\text {Ind }}$ cells treated with doxycycline required to eliminate 189 completely the asymmetry in competition, reducing the competition strength that $p 53$ mutant 190 cells have on $\mathrm{Bcl}^{\text {Ind }}\left(k_{W P}\right)$ to the same level than homotypic competition $\left(k_{W W}=k_{W P}\right)$ (Fig. 191 3I). Interestingly, it also required to eliminate the asymmetry in the intrinsic population 192 growth by increasing the net proliferation rate of $B c / 2^{\text {Ind }}$ cells to the same level as $p 53^{-/}$ 193 ESCs $\left(\rho_{W}=\rho_{P}\right)$. In summary, BCL2 rescue dynamics could only be reproduced by the 194 model when all the parameters of wild-type cells are restored to the equivalent parameter of $195 p 53^{-}$ESC, supporting the hypothesis that the competition mechanism is an active 196 elimination of wild-type cells by $p 53^{-/}$cells.

197 Short range signalling mediates the elimination of wild-type cells by $p 53^{/-}$ 198 ESCs.

199 The data presented in Figs. 1-3 points to a direct competition between $p 53^{-/}$and wild-type 200 cells, whereby one induces the elimination of the other. But in addition to the direct 201 competition model (Eq. (1)) we also wanted to explore the possibility that cells compete for a 202 shared resource that is consumed over time with differential rates and tolerances depending 203 on the cell type (see Materials and Methods, Eq. (3)). Interestingly, this model was not able 204 to recapitulate the experimental growth curves (Fig. 2E-F and Fig. S3). To test this 205 assumption experimentally we analysed the effect that providing unlimited amounts of 206 nutrients has on the dynamics of the competition between wild-type and $p 53^{-/}$cells. For this 207 we performed our competition experiments in a culture media that was continuously 208 perfused from day 1 of culture by a pump (Fig. 4A). Using this system, we observed that 209 both wild-type and $p 53^{-/}$ESCs grew roughly double as much when the media was perfused 
210 compared to when it wasn't, in both separate and co-culture conditions (Fig. 4B). This

211 increase in growth was likely due to a lower level of cell death, as the percentage of cells

212 that were positive for cleaved caspase 3 was also significantly reduced in the perfusion

213 condition (Fig. 4C). It is also possible that the apoptotic cells are being more efficiently

214 removed due to the perfusion. Notably, we found that despite these lower levels of

215 apoptosis, the perfusion did not significantly affect the degree to which wild-type ESC were

216 eliminated in co-culture (Fig. 4D)., suggesting that the latter may be true. These results

217 suggest two things. First, they indicate that the elimination of wild-type cells by $p 53^{-/} \mathrm{ESCs}$ is

218 unlikely to be due to nutrient depravation. Second, the fact that the number of cells in the

219 culture dish can double without increasing the degree of wild-type apoptosis, also suggests

220 that this elimination is not due to mechanical stress, as has been shown to be the case for

221 MDCK cells with increased p53 expression (Wagstaff et al., 2016).

222 We next analysed the possibility that diffusible growth factors secreted into the media could 223 be inducing the elimination of loser cells. For this we did two things. First, we analysed the 224 effect of conditioned media taken from wild-type and p53 mutant cells cultured separately, 225 as well as well as taken from their co-culture. We observed that none of these conditioned 226 medias had any effect on the growth of wild-type cells and they all reduced $p 53^{-/}$cell growth 227 (Fig. 4E). To further address the importance of secreted factors we used a fences system 228 (Lawlor et al., 2020), whereby one population of cells is grown surrounded by another but 229 separated by fences that are removed once the cells are seeded (Fig. 4F). This allows the 230 different cell populations to be cultured without contact but sharing the same media. When 231 this was done, we observed that cells grown on the outside layer grew slower than those 232 cultured on the inside, irrespective of their genotype (Fig. 4G-H). Importantly, we found that 233 wild-type cells grew similarly if they were surrounded without contact by p53 mutant cells or 234 by other wild-type cells (Fig. 4I). Together this data suggest that cell-cell contact or short235 range signalling is required for $p 53^{-/}$cells to eliminate wild-type cells.

\section{Cell neighbourhood changes during cell competition}

237 Given the importance of short-range signalling for the elimination of wild-type cells, we 238 asked if changes in cell neighbourhood could explain the competition between wild-type and $239 p 53$ mutant cells. We consider here the local cell neighbourhood to be the relative numbers 240 of wild-type and $p 53^{-}$cells that are in direct contact with a given cell in the co-culture 241 condition. When we analysed the cell neighbourhood of wild-type cells, we found that during 242 the time-course of the experiment these cells increased the average number of wild-type 243 neighbours until reaching a peak at day 2 of co-culture, but that after this time-point this 244 number of wild-type neighbours decreased (Fig. 5A-B). In contrast to this, the average 245 number of $p 53^{-/}$neighbours that wild-type cells had increased from day 1 and by day 3 they 
246 had more $p 53^{-/}$neighbours than wild-type neighbours. This switch in wild-type

247 neighbourhood between days 2 and 3 coincided with when wild-type elimination is most

248 obvious in the growth curves of these cells (Fig. 1C). The same neighbourhood dynamics

249 could be observed for Bcl $2^{\text {Ind }}$ cells when these were co-cultured with $p 53^{-/}$ESCs without

250 doxycycline (Fig. 5C). However, if BCL2 is induced with doxycycline and loser cell

251 elimination is prevented, then $\mathrm{Bcl} / 2^{\prime n}$ cells showed a daily increase in $\mathrm{Bcl} / 2^{\text {In }}$ cell neighbours,

252 while the number of $p 53^{-/}$ESC neighbours first increased until day 2 and then decreased

253 (Fig. 5D). These results are consistent with cell neighbourhood having a role in the outcome 254 of competition.

255 We next tested the effects of changing the concentrations of cells plated. First, we analysed 256 the effects of reducing the cell numbers plated. For this a mix of $0.08 \times 10^{5}$ wild-type cells and $2570.08 \times 10^{5} p 53^{-/}$ESCs were plated in the co-culture condition. We observed that at this low 258 confluency there was no substantial change in the neighbourhood of wild-type cells in co259 culture (Fig. 5E). Interestingly, this correlated with the total number of wild-type cells still 260 increasing at day 4 of co-culture (Fig. S5A). This suggests that the low confluency is 261 reducing the number of possible $p 53^{-/}$neighbours, and that this is decreasing the rate of 262 competition. We then analysed the effects of increasing the cells numbers plated at day 0 to 263 a mix of $5 \times 10^{5}$ wild-type and $5 \times 10^{5} p 53^{-/}$cells in co-culture. This condition led to wild-type 264 cells having more $p 53^{-/}$cell neighbours from day 2 (Fig. $5 \mathrm{~F}$ ) and being robustly eliminated in 265 co-culture (Fig. S5B). Similar was observed when we changed the proportion of wild-type to $266 p 53^{-/}$cells plated. When a mix of $2 \times 10^{5}$ wild-type and $1 \times 10^{5} p 53^{-/}$cells were plated in co267 culture this caused a shift between days 2 and 3 in the neighbourhood of wild-type cells from 268 having more wild-type neighbours to having more $p 53^{-/}$ones (Fig. 5G). This correlated with 269 when the wild-type cells were eliminated in co-culture (Fig. S5C). These results, together

270 with the importance of short-range signalling identified in Fig. 4, point to the direct interaction 271 between wild-type and $p 53^{-/}$cells determining the outcome of their competition. Our findings 272 also suggest that the relative number of neighbours with a different competitive ability is a 273 factor affecting the replacement of wild-type cells.

\section{DISCUSSION}

275 Cell competition is a fitness quality control mechanism that eliminates cells that are less-fit 276 than their neighbours. One important implication of cell competition is that the fitness of a 277 cell is relative to the fitness of its neighbour. For example, in mouse, wild-type cells eliminate 278 cells with mitochondrial dysfunction (Lima et al., 2021), but are eliminated by cells that over279 express c-MYC (Claveria et al., 2013; Sancho et al., 2013). Here, we have addressed how 280 the expression levels of P53 affects the competitive nature of pluripotent cells. We find that 
281 cells lacking $p 53$ behave as super-competitors and eliminate their wild-type neighbours. This

282 elimination is dependent on the onset of differentiation, as when cells are cultured in

283 pluripotency conditions mutation of p53 provides cells with a competitive advantage.

284 Furthermore, by combining mathematical modelling and cell-based assays we also show

285 that the competitive advantage of $p 53$ null mutant cells is mediated by short-range signalling

286 interactions that induce the elimination of wild-type cells, rather than through a competition

287 for nutrients or space. This highlights the importance of the local cell neighbourhood for the

288 regulation of proliferation during early embryonic development.

289 Over the last few years there has been increasing evidence for a key role for P53 in cell

290 competition. In Drosophila p53 has been shown to be required in Myc-overexpressing

291 super-competitor cells to induce wild-type cell elimination (de la Cova et al., 2014). In these

292 winner cells, p53 regulates metabolism by promoting oxidative phosphorylation and

293 inhibiting glycolysis. In contrast to this, mechanical stress induces increased $p 53$ expression

294 in polarity deficient MDCK cells and this activation causes the elimination of these loser cells

295 (Wagstaff et al., 2016). In mouse cell competition, p53 has been shown to play multiple

296 roles. In haemopoietic stem/progenitor cells, DNA damage causes the out-competition of

297 cells with higher p53 levels (Bondar and Medzhitov, 2010). Here the damage induces

298 senescence in those cells that have higher p53 levels than their neighbours. In the mouse

299 embryo it was found that clones carrying a mutation of $M d m 2 / 4$, and therefore that have

300 elevated P53 expression, are outcompeted by wild-type cells (Zhang et al., 2017). Similarly,

301 we have shown that during the onset of differentiation, defective cells such as cells with

302 impaired BMP signalling or tetraploid ESCs, show increased p53 expression that is required

303 for their out-competition by wild-type cells. We also demonstrated that the mechanism of

304 elimination of these defective cells is because in a competitive environment p53 represses

305 mTOR signalling and this induces apoptosis. Our results, together with these studies,

306 suggests therefore that p53 could act as a general sensor of cell fitness across different 307 tissues.

308 Three main modes of cell competition have been proposed, competition for nutrients, 309 mechanical competition, and direct fitness sensing between cells (Bowling et al., 2019). For

310 example, during cell competition in MDCK cells, elevated p53 expression sensitises cells to

311 compaction, indicating that differences in p53 expression determine a differential response

312 to mechanical stress (Wagstaff et al., 2016). In Drosophila, p53 regulates cell metabolism to

313 determine the competitive nature of cells in the imaginal wing disc (de la Cova et al., 2014).

314 This metabolic role could be used to infer that p53 is required to establish differences in

315 nutrient metabolism that will in turn direct the outcome of competition. But our studies

316 described here analysing the behaviour of $p 53$ mutant pluripotent cells suggest that during 
317 ESC differentiation p53 is possibly playing roles that are different to those described above

318 for MDCK cells and in Drosophila. Our observation that there is no change to the cell

319 competition dynamics between wild-type and $p 53^{-}$ESCs despite cell numbers doubling

320 when assays are performed in media that is being continuously replenished through cell

321 perfusion, suggests that neither nutrient or space availability is determining the outcome of

322 the competition between these cell types. Instead, our findings reveal that a change in the

323 cell neighbourhood of loser wild-type cells is correlated with the outcome of cell competition.

324 This suggests that the local interaction between wild-type and mutant cells regulates the

325 elimination of the wild-type cells. These results are in line with what has been observed with

326 c-Myc over-expressing ESCs, that eliminate wild-type cells via short-range/contact

327 dependant signalling (Diaz-Diaz et al., 2017) and contrast with our own findings that the

328 elimination of ESCs with defective BMP signalling is mediated by long-range signalling

329 (Sancho et al., 2013). Our observations that the relative number of winner/loser neighbours

330 determines the outcome of cells competition are also in accordance with what has been

331 found in the Drosophila pupal notum, where the super-competition ability of $c$-Myc over-

332 expressing cells has been related to the relative surface area shared between winner and

333 loser cells (Levayer et al., 2015). This suggests that replacement of wild-type cells in a

334 tissue occurs through a different mechanism than the replacement of dysfunctional cells,

335 and therefore during embryonic development there may be several distinct forms of cell

336 competition acting in a tissue at the same time.

337 Computational and mathematical modelling has provided invaluable insight allowing us to

338 distinguish between possible models of competition, as well as revealing and quantifying the

339 minimal rules required to reproduce the competition dynamics observed. Further

340 descriptions of local competition in confluent populations - where the population cannot be

341 considered homogeneous - will require a mathematical framework that incorporates the

342 spatial dynamics of the competition. Such models can use similar ODE formulations where

343 clone shape is taken into account implicitly in the functional form of the competition terms

344 (Nishikawa et al., 2016). Alternatively, more detailed descriptions of the cellular monolayer

345 can be simulated by using a computational vertex model, where the dynamics and

346 environment of each individual cell is taken into account. These spatial models can allow us

347 to study additional modes of competition resulting from dynamics exclusive to confluent

348 tissues. For example, interaction between cell populations growing at different rates has

349 been shown to introduce a mechanical stress that can act as a feedback mechanism to

350 stabilize uniform growth (Shraiman, 2005). Similarly, cell geometry and mechanical

351 heterogeneities within a developing tissue can bias mechanical cell elimination leading to

352 effective changes in competition properties of coexisting cellular populations (Lee and 
353 Morishita, 2017). In addition, following cell elimination, cell-type specific topological

354 remodelling of epithelial junctions has been shown to be enough to induce a difference in the

355 fitness between cell populations in the Drosophila wing disc (Tsuboi et al., 2018).

356 More sophisticated agent-based multi-scale models can be used to simulate more specific 357 cell and tissue morphologies, such as competition during the transition to congruence. An 358 example of such models was used in (Gradeci et al., 2021) using automatic annotation of 359 movies of co-cultured wild-type and polarity deficient MDCK cells lasting up to 4 days, 360 providing extensive input on the behaviour of wild-type and mutant cells. Their modelling 361 identified that cell density and stiffness is sufficient to account for the apoptotic elimination of 362 loser cells during mechanical competition. In contrast to this, the outcome of biochemical 363 competition appears to be primarily regulated by the organization of winner and loser cells in 364 the tissue. Interestingly, these results are very much in accordance with the likely role of cell 365 neighbourhood identified in our study. One of the main limitations of the successful 366 application of spatial computational models is the large amount of parameters and rules that 367 can be used to describe the behaviour of cellular populations, making hard to infer 368 confidently details of the model even in simple scenarios (Kursawe et al., 2018). This 369 highlights the necessity of minimal models able to incorporate and test mechanistic 370 hypothesis matching the complexity of the available data.

371 In conclusion, our studies identify that upon exit from pluripotency, loss of p53 expression is 372 sufficient to make ESCs into super-competitors. These winner cells induce the replacement 373 of healthy wild-type cells via a direct induction of apoptosis. This cell replacement not only 374 provides a potential explanation for the expansion of cells with p53 mutations in human 375 pluripotent stem cell cultures (Merkle et al., 2017), but importantly together with our finding 376 that those cells eliminated in the early mouse embryo have a signature of elevated p53 377 expression (Lima et al., 2021), suggest that competitive interactions between cells with 378 different levels of p53 expression shape growth during development.

\section{MATERIALS AND METHODS}

\section{Cell line generation}

381 To generate $p 53^{-/}$cells, a p53 sgRNA (5'-GCAGACTTTTCGCCACAGCG-3') was cloned 382 into lentiCRISPRv2 vector. Viruses were generated by transfecting this vector along with 383 helper plasmids VSV-G and psPAX2 into HEK293T packaging cells. After $48 \mathrm{~h}$, the media 384 from these cells was applied to ESCs with $4 \mu \mathrm{g} / \mathrm{ml}$ polybrene. Two rounds of infection, for $4 \mathrm{~h}$ 385 and then overnight, were carried out. The cells were then selected using $2 \mu \mathrm{g} / \mathrm{ml}$ puromycin 386 and plated at single cell confluency. Clones were screened for loss of p53 protein by 387 western blot. 
388 To generate p21\% cells, a p21 sgRNA (5'- GATTGCGATGCGCTCATGGC-3') was cloned 389 into the px330 vector (Addgene). ESCs were co-transfected with $2 \mu \mathrm{g}$ of this vector and $390 \quad 0,12 \mu \mathrm{g}$ of an hygromycin marker (\#631625, TAKARA) using Lipofectamine 2000 (Invitrogen) 391 according to manufacturer's instructions. The cells were selected using $\mathrm{x \mu g} / \mathrm{ml}$ hygromycin 392 and plated at single cell confluency. Clones were screened for loss of p21 protein by 393 western blot.

394 To generate ${ }^{B c / 21 n d}$ cells, ESCs were co-transfected with $1 \mu \mathrm{g} \mathrm{pPB-TRE(3G)-hBCL2-PURO,}$ $3951 \mu \mathrm{g}$ pPB-CAG-rtTA(3G)-NEO and $1 \mu \mathrm{g}$ pCMV-PBase using Lipofectamine 2000 (Invitrogen) 396 according to manufacturer's instructions. The cells were selected using $1 \mu \mathrm{g} / \mathrm{ml}$ doxycycline,

$3972 \mu \mathrm{g} / \mathrm{ml}$ Puromycin and $x \mu \mathrm{g} / \mathrm{ml}$ Neomycin and plated at single cell confluency. Clones were 398 screened by western blot for BCL2 protein overexpression upon $1 \mu \mathrm{g} / \mathrm{ml}$ doxycycline 399 treatment.

\section{Cell culture}

401 All cells were cultured at $37^{\circ} \mathrm{C}$ in an atmosphere with $5 \% \mathrm{CO} 2$. Reagents used for tissue 402 culture were obtained from Invitrogen unless otherwise stated. Mouse embryonic stem cells 403 (ESCs) were cultured on $0.1 \%$ gelatin-coated flasks (Nunc, Thermo Fisher) in GMEM 404 containing with $10 \%(\mathrm{v} / \mathrm{v}$ ) foetal calf serum (FCS; Seralab), 1X non-essential amino acids, 2 $405 \mathrm{mM}$ L-glutamine, $0.1 \mathrm{mM} \beta$-mercaptoethanol and supplemented with homemade leukaemia 406 inhibitory factor (1:1000, LIF). ES cells were routinely dissociated with trypsin and 407 cryopreserved in 10\%DMSO in FCS.

\section{Competition assay}

409 Cells were seeded onto plates coated with fibronectin (Merk) at a concentration of $2.5 \times 10^{4}$ 410 cells $/ \mathrm{cm}^{2}$ either separately or mixed for co-cultures at a 50:50 ratio (Sancho et al., 2013). 411 Cells were cultured in N2B27 media (Neurobasal media; DMEM F12 media, $0.5 \times$ B27 412 supplement; 0.5 x N2 supplement; $0.1 \mathrm{mM}$ 2-mercaptoetanol; $2 \mathrm{mM}$ glutamine; all Thermo 413 Fisher Scientific) during 3-4 days to allow for differentiation. At the indicated time points, the 414 cells were counted using Vi Cell Counter and Viability Analyser (Beckman Coulter) and 415 proportions of each cell type in co-cultures were determined using LSR II Flow Cytometer 416 (BD Bioscience). Sytox blue (Thermo Fisher Scientific) or propidium iodide (Sigma) was 417 used to stain for dead cells.

\section{Perfusion assay}

419 Cells were seeded into 6-well perfusion plates (AVP011, Reprocell) coated with fibronectin 420 either separately or mixed for co-cultures at a 50:50 ratio in N2B27 media. At day 1, the 421 plate was connected to a bottle with $200 \mathrm{ml} \mathrm{N2B27}$ and perfused using a Watson Marlow $422120 \mathrm{U}$ Pump with a speed of $9 \mathrm{pm}$. The media circulated from the bottle to the plate, where 
423 each well is connected to the next one by a channel, so a unidirectional flow of media is

424 established to each well, and then back to the bottle. The culture was perfused until day 4,

425 when the cells were counted or fixed.

426 Fences and conditioned media assays

427 For Fence assays fences (Aix-Scientifics) were placed in each well of a 24-well plate coated

428 with fibronectin. $0.008 \times 10^{6}$ cells were seeded in the inner ring and $0.035 \times 10^{6}$ cells in the

429 outer ring. The fences were removed the following day and the media was replaced every

430 day. At the indicated time points the fences were replaced into the well to count cell numbers

431 using Vi Cell Counter and Viability Analyzer (Beckman Coulter).

432 For conditioned media assays, $0.08 \times 10^{6}$ Wild-type or $p 53^{-/}$cells were seeded and from day

4332 cells were cultured with conditioned media obtained from wild-type or $p 53^{-\%}$ cells cultured

434 separately or from a co-culture. Conditioned media was obtained from the corresponding cell

435 types and was concentrated using Vivaspin 500 centrifugal concentrators (G E Healthcare)

436 according to manufacturer's instructions.

\section{$437 \quad$ Flow cytometry staining}

438 Cells were detached from the plates using accutase (Sigma) and fixed in 7.4\% formaldehyde

439 in N2B27 media for 10 minutes. Permeabilization was carried out using ice-cold methanol 440 and cells were blocked using 1\% BSA. Cells were then incubated with primary antibody 441 (Cleaved Caspase 3 \#9664, Cell signaling,1:200 dilution) for 1 hour at room temperature. 442 After washing, cells were incubated with the secondary antibody (Alexafluor-546/405, 443 Thermo Fisher Scientific, 1:2000 dilution) for 30 minutes at room temperature. Flow 444 cytometry was performed using LSR II Flow Cytometer and analysed using FlowJo software 445 v9 or v10.0.7r2 (BD Bioscience).

\section{Proliferation assay}

447 Cells were incubated with EDU from the Click-iT kit (Thermo Fisher Scientific) for 2 hours 448 according to manufacturer's instructions. Cells were then detached from plates using 449 accutase and analysed by flow cytometry using LSR II Flow Cytometer and FlowJo software.

\section{Stem cells immunofluorescence}

451 For immunostaining ESCs and EpiSCs were fixed for 10min in 4\%PFA at room temperature, 452 permeabilised in $0,4 \%$ Triton-X100/PBS for 5 minutes at room temperature, blocked in $45310 \% \mathrm{BSA} / 0,1 \%$ Triton X-100/PBS and incubated overnight at $4^{\circ} \mathrm{C}$ in primary antibody diluted 454 in 1\%BSA/0,1\%Triton X-100 (anti-Cleaved Caspase 3 Asp175, Cell Signalling, 1/100; anti455 Nanog (14-5761-80 eBioscience, 1/100, anti ATP-b (Ab14730, Abcam - 1:200). Alexa-Fluor 456 conjugated secondary antibodies (Thermo Fisher Scientific) were used at 1/500 dilution in $4571 \% \mathrm{BSA} / 0,1 \%$ Triton $\mathrm{X}-100$. Cells were mounted for visualization in Vectashield with DAPI 
458 (Vector Laboratories). Images were acquired with a Zeiss confocal microscope and analysed

459 with the Fiji software (Schindelin et al., 2012).

\section{Western blot analysis}

461 Cell lysates were collected in Laemmli buffer $(0.05 \mathrm{M}$ Tris- $\mathrm{HCl} \mathrm{pH} 6.8,1 \% \mathrm{SDS}, 10 \%$ 462 Glycerol, $0.1 \% \beta$-mercaptoethanol) and denatured for 10 minutes at $95^{\circ} \mathrm{C}$, quantified using 463 BCA quantification (Thermo Fisher Scientific) and resolved using CriterionXT pre-cast gels 464 (BioRad) and transferred to nitrocellulose membranes. Blocking was performed in $5 \%$ milk in 465 TBST buffer and primary antibody incubation (p53 \#2524, p21, Bcl2, Tubulin) was done 466 overnight at $4^{\circ} \mathrm{C}$ in TBST containing 5\% BSA. Western blot quantification was performed 467 using Fiji software version 2.0.0-rc-49/1.51d. Protein expression levels were normalized to 468 loading control tubulin.

\section{RNA Extraction and Quantitative RT-PCR}

470 Total RNA was extracted with the RNeasy mini kit (Qiagen) and SuperScript III reverse 471 transcriptase (Thermo Fisher Scientific) was used for cDNA synthesis according to 472 manufacturer's instructions. Quantitative RT-PCR was performed by amplification with 473 SYBR green Master Mix (Roche). The primers used are listed in Table 1. RNA samples from 474 wild type and mutant clones were collected from 3 independent experiments.

475

476 Table 1: Primers used in the quantitative RT-PCR

\begin{tabular}{|ccc|}
\hline Gene & \multicolumn{1}{c|}{ Forward } & Reverse \\
\hline Gapdh & 5' CATGGCCTTCCGTGTTCCTA 3' & 5' GCGGCACGTCAGATCCA 3' \\
\hline Fgf5 & 5' AAAGTCAATGGCTCCCACGAA 3' & 5' CTTCAGTCTGTACTTCACTGG 3' \\
\hline Esrrb & 5' GGACACACTGCTTTGAAGCA 3' & 5' ACAGATGTCTCTCATCTGGC 3' \\
\hline Nanog & 5' CTTACAAGGGTCTGCTACTGAGATGC 3' & 5' TGCTTCCTGGCAAGGACCTT 3' \\
\hline Rex1 & 5' CGAGTGGCAGTTTCTTCTTGG 3' & 5' GACTCACTTCCAGGGGGCAC 3' \\
\hline
\end{tabular}

477

\section{Statistical methods}

479 Statistical analysis was performed using GraphPad Prism v8.0.0 software. Statistical

480 methods used are indicated in the relevant figure legends. No randomization or blinding was 481 used in experiments. Sample sizes were selected based on the observed effects and listed 482 in the figure legends. Statistical significance was considered with a confidence interval of $4830.05 \% ;{ }^{*} p<0.05 ;{ }^{* *} p<0.01 ;{ }^{* *} p<0.001 ;{ }^{* * *} p<0,0001$.

484 Data obtained from cell competition assays were analyzed by two-way ANOVA, followed by 485 Holm-Sidak's multiple-comparison test. Data obtained from RT-PCR were analyzed by one- 
486 way ANOVA followed by Turkeys post-hoc test. Data obtained from western blot were 487 analyzed with an unpaired two tailed $t$-test.

\section{Model simulation and Approximate Bayesian inference}

Ordinary differential equations where solved with a custom explicit forward Euler method. Parameter distributions compatible with the model were inferred using a Markov chain Monte Carlo implemented in the multiple-try differential evolution adaptive Metropolis algorithm PyDREAM (Shockley et al., 2018). The log-likelihood function for the parameter set $\theta$ assumed that experimental trajectories deviations $\left(W_{\text {exp }}, P_{\text {exp }}\right)$ followed a Gaussian distribution centred at each timepoint of the observed trajectories $\left(W_{\text {model }}, P_{\text {model }}\right)$ with a width given by the square root of the population size,

$$
\log \mathcal{L}(\text { data } \mid \theta)=-\sum_{j=1}^{M} \sum_{i=1}^{N_{j}}\left(\left(\frac{W_{\text {exp }}^{j, i}-W T_{\text {model }}\left(X_{j}^{0}, \theta, t_{i}\right)}{\sqrt{W_{\text {exp }}^{j, i}}}\right)^{2}+\left(\frac{P_{\text {exp }}^{j, i}-P_{\text {model }}\left(X_{j}^{0}, \theta, t_{i}\right)}{\sqrt{P_{\text {exp }}^{j, i}}}\right)^{2}\right) \cdot \quad \operatorname{Eq}(2)
$$

Where the index $j$ runs for all the experimental initial conditions $X_{j}^{0} \equiv(W, P)_{t=0}$; and the index $i$ for all the replicates and their time points $t_{i}$. Convergence of the posterior parameter distributions was assessed using the Gelman Rubin diagnostic with a threshold $R_{c}=1.2$ across 5 different sampling chains.

\section{Resource competition model}

504 The scenario in which cells compete for an external resource was modelled introducing an 505 additional chemical species $R$ that is depleted with cell specific rates $\gamma_{W}$ and $\gamma_{P}$. The 506 nutrient reduces the intrinisic apoptotic rate of each cell species $\delta_{W}$ and $\delta_{P}$ depending on the 507 characteristic concentrations $r_{W}$ and $r_{P}$.

$$
\begin{aligned}
& \frac{\mathrm{d} W}{\mathrm{~d} t}=W\left(\alpha_{W}-\frac{\delta_{W} r_{W}}{r_{W}+R}\right), \\
& \frac{\mathrm{d} P}{\mathrm{~d} t}=P\left(\alpha_{P}-\frac{\delta_{P} r_{P}}{r_{P}+R}\right), \\
& \frac{\mathrm{d} R}{\mathrm{~d} t}=-H(R)\left(\gamma_{W} W+\gamma_{P} P\right) .
\end{aligned}
$$

508 Where $H(R)$ is the Heaviside step function.

\section{ACKNOWLEDGEMENTS}

510 We would like to thank all members of the molecular embryology group for their technical

511 and scientific support, especially Dr. Aida di Gregorio and Dr. Ana Lima. We would also like

512 to thank Stephen Rothery for guidance and advice with confocal microscopy. We thank

513 James Elliot and Bhavik Patel from the LMS/NIHR Imperial Biomedical Research

514 Centre Flow Cytometry Facility for support. 


\section{COMPETING INTERESTS}

516 The authors declare no competing interests.

\section{FUNDING}

518 Research in Tristan Rodriguez lab was supported by the MRC project grants 519 (MR/N009371/1 and MR/T028637/1) and by the BBSRC project grant (BB/S008284/1).

520 Salvador Perez was supported by a Commission of the European Communities H2020

521 MSCA IF fellowship (709010) and by a long-term EMBO post-doctoral fellowship. The

522 Facility for Imaging by Light Microscopy (FILM) at Imperial College London is part-supported

523 by funding from the Wellcome Trust (grant 104931/Z/14/Z) and BBSRC (grant 524 BB/L015129/1). 


\section{FIGURE LEGENDS}

526 Figure 1. Wild-type cells are outcompeted by $p 53^{-/}$cells. A. p53 levels in wild-type and 527 two different $p 53^{-}$clones untreated or treated with the p53 activator Nutlin-3a for $4 \mathrm{~h}$. B. 528 Growth curves of wild-type and $p 53^{-/}$cells over 4 days in separate or co-culture in 529 pluripotency conditions. C. Fold change in wild-type and $p 53^{-/}$cell numbers between day 0 530 and day 4 when cultured alone or co-cultured in pluripotency conditions. D. Growth curves of 531 wild-type and $p 53^{-/}$cells over 4 days in separate or co-culture differentiation conditions. E. 532 Fold change in wild-type and $p 53^{-/}$cell numbers between day 0 and day 4 when cultured 533 alone or co-cultured in differentiation conditions. F. Quantitative RT-PCR showing gene 534 expression levels of naïve and primed pluripotency markers in wild-type and $p 53^{-/}$ESCs in 535 pluripotency and differentiation. Gene expression is normalized against beta-Actin. G. 536 Percentage of EDU incorporation in wild-type cells and $p 53^{-/}$cells cultured for 4 days 537 separately or co-cultured. $\mathbf{H}$. Percentage of cleaved caspase 3 positive cells in wild-type and $538 p 53^{-/}$cells cultured for 3 and 4 days separately or co-cultured. I. Levels of Cleaved Caspase 5398 in Wild-type and $p 53^{-/}$cells cultured alone or co-culture for 3 days. Data were obtained 540 from three independent experiments and are shown as the mean + SEM (B-I).

541 Figure 2. A mathematical model suggests a direct asymmetric competition. A 542 Schematic depicting how the net proliferation rate per cell in a homotypic population 543 depends on cell density. B Schematic showing the four possible direct competition 544 mechanisms affecting population growth of WT and $p 53^{-/}$cells. C Comparison of the 545 experimental data (one circle per replicate) with the model prediction (lines) for the direct 546 competition model (Eq. 1) for 9 of 24 the initial conditions used in the inference (the rest can 547 be found in Fig. S3). Shaded zones show model prediction for the inferred parameter region 548 with likelihood $>90 \%$ of its maximum. Parameter inference values can be found in Fig. S4. D 549 Inferred credibility distributions for the relative differences between different model 550 parameters. Relative differences are significant between the intrinsic growths and between 551 the competition constants on WT cells. E Log-likelihood distributions of the direct 552 competition model (Eq. 1) and the resource competition model (Eq. 3). F Comparison of the 553 experimental data with the resource competition model, visualization details are the same as 554 in panel C. Parameter inference values can be found in Fig. S4.

555 Figure 3. p53 $^{-/}$ESCs induce the apoptotic elimination of wild-type cells in coculture. 556 A. Bcl2 levels in wild-type and $B c / 2^{I N D}$ cells not treated or treated with doxycycline for 3 days.

557 B. Percentage of cleaved caspase 3 positive cells in $B c / 2^{I N D}$ treated with or without 558 doxycycline and $p 53^{-/}$cells. C. Percentage of EDU incorporation in $B c / 2^{I N D}$ treated with or 559 without doxycycline and $p 53^{-/}$cells. D. Growth curves of $B c / 2^{I N D}$ and $p 53^{-/}$cells over 4 days 560 in separate or co-culture conditions without doxycycline treatment. E. Fold change in wild561 type and $p 53^{-/}$cell numbers between day 0 and day 4 . F. Growth curves of $B c / 2^{I N D}$ and $p 53^{-/}$ 
562 cells over 4 days in separate or co-culture conditions with doxycycline treatment from day 1.

563 G. Fold change in wild-type and $p 53^{-/}$cell numbers between day 0 and day 4 . Data were 564 obtained from three independent experiments and are shown as the mean + SEM (E-H). $\mathbf{H}$ 565 Comparison of the direct competition model (lines) with the experimental data (one replicate 566 per circle) for doxycycline treatment at different days (vertical dashed lines). Shaded zones 567 show model prediction for the inferred parameter region with likelihood $>90 \%$ of its 568 maximum. Parameters are the same as in Figs. 2, S3, S4. I Resulting model trajectories 569 when doxycycline treated cells are simulated by only changing the intrinsic growth and 570 maintaining differences in competition strength (left), or only changing the competition 571 strength and maintaining differences in intrinsic growth (right).

572 Figure 4. Wild-type cell elimination is cell contact dependent. A. Schematic 573 experimental setup whereby cells were cultured separately and together and N2B27 media 574 was perfused over the plate from day 2 until day 4 . Cells were then counted and fixed. B. 575 Wild-type and $p 53^{-/}$cell numbers over 4 days cultured separately or together in a control 576 plate and in a perfused plate. C. Fold change in wild-type cell numbers between day 0 and 577 day 4 when cultured alone or with $p 53^{-/}$cells in N2B27 in a control plate and in a perfused 578 plate. D. Percentage of cleaved caspase 3 in wild-type and $p 53^{-/}$cells cultured separately or 579 in co-culture with and without perfusing N2B27 media. E. Cell numbers of wild-type and p53

580 / cells cultured separately for 4 days and adding conditioned media from day 3 to day 4 . 581 Conditioned media was taken from cultured wild-type and $p 53^{-/}$cells or from a co-culture of 582 both cells types. F. Schematic experimental setup whereby wild-type cells where cultured 583 surrounded by $p 53^{-/}$cells or wild-type cells in the same well without cell contact between 584 both cell types. G. Growth curves of wild-type and $p 53^{-/}$cells over 4 days cultured as in F. H. 585 Fold change in wild-type and $p 53^{-/}$cell numbers between day 0 and day 4 . I. Fold change in 586 wild-type cell numbers a between day 0 and day 4 when they are cultured surrounded by 587 wild-type or $p 53^{-}$cells. Data were obtained from three independent experiments and are 588 shown as the mean + SEM (A-I).

589 Figure 5. Neighbourhood changes during cell competition. A. Immunostainings of wild590 type and $p 53^{-/}$cells co-cultured from day 1 to day 4 of the culture. An example of neighbours 591 of wild-type cells is shown for each day. B. Number of wild-type and $p 53^{-/}$neighbours of 592 wild-type cells from day 1 to day 4 of the co-cultures. C. Number of $B c / 2^{I N D}$ and $p 53^{-1-}$ 593 neighbours of $B c / 2^{I N D}$ cells from day 1 to day 4 of co-cultures without doxycycline treatment. 594 D. Number of $B c / 2^{I N D}$ and $p 53^{-/}$neighbours of $B c / 2^{I N D}$ cells from day 1 to day 4 of co-cultures 595 with doxycycline treatment from day 1 . E. Number of wild-type and $p 53^{-/}$neighbours of wild596 type cells from day 1 to day 4 of co-cultures with lower initial cell numbers plated. F. Number 597 of wild-type and $p 53^{-/}$neighbours of wild-type cells from day 1 to day 4 of co-cultures with 598 higher initial cell numbers plated. G. Number of wild-type and $p 53^{-/}$neighbours of wild-type 
599 cells from day 1 to day 4 of co-cultures with different percentage of seeded cells. Data were

600 obtained from three independent experiments and are shown as the mirrored distribution of

601 wild-type and $p 53^{-/}$neighbour numbers (B-G).

602 Figure S1: A. P21 levels in wild-type and p21\% clones untreated or treated with the P53 603 activator Nutlin-3a for 4h. B. Quantitative RT-PCR showing gene expression levels of naïve 604 and primed pluripotency markers in wild-type and $p 21^{-}$ESCs in pluripotency and 605 differentiation culture conditions. Gene expression is normalized against beta-Actin. C. 606 Growth curves of wild-type and $p 21^{-}$cells cultured for 4 days in separate or co-culture 607 differentiation conditions. D. Fold change in wild-type and $p 21^{-/}$cell numbers between day 0 608 and day 4 when cultured separately or co-cultured. E. Percentage of EDU incorporation in 609 wild-type and $p 21^{-1 /}$ cells at day 4 cultured separately or co-cultured in differentiation 610 conditions. Data were obtained from three independent experiments and are shown as the 611 mean + SEM (B-E).

612 Figure S2. A. Levels of un-cleaved Caspase 8 and its cleaved forms p41 and p18 in wild613 type and $p 53^{-/}$cells cultured separately for 3 days in differentiation conditions. B. 614 Quantification of a. C. Immunostainings of wild-type and $p 53^{-/}$cells cultured in separate 615 conditions or co-cultured for 3 days showing an increase in Cleaved Caspase 8 in wild-type 616 cells. D Fold change in wild-type and $p 53^{-/}$cell numbers between day 0 and day 4 when 617 cultured in separate conditions or co-cultured in differentiation conditions and treated with 618 DMSO or pan caspase inhibitors from day 3 to day 4. Data were obtained from three 619 independent experiments and are shown as the mean + SEM (A-D).

620 Figure S3. Comparison of the experimental data (one circle per replicate) with the model 621 prediction (lines) for the direct competition model (Eq. 1) for all the different 24 experimental 622 plating conditions used in the inference. Shaded zones show model prediction for the 623 inferred parameter region with likelihood $>90 \%$ of its maximum. Parameter inference values 624 can be found in Fig. S4.

625 Figure S4. Posterior credibility distributions for the direct competition model (Eq. 1) (A), and 626 for the resource competition model (B). Diagonal panels show marginal posterior distribution 627 histograms. Off-diagonal panels show the sampled pairwise joint distribution for all the 628 possible parameter pairs. Colour indicates magnitude of the likelihood.

629 Figure S5. A. Growth curves of wild-type and $p 53^{-/}$cells cultured for 4 days in separate or 630 co-culture conditions with a starting cell number of $0.016 \times 10^{6}$ cells seeded per well. B. 631 Growth curves of wild-type and $p 53^{-/}$cells cultured for 4 days in separate or co-culture 
632 conditions with a starting cell number of $1 \times 10^{6}$ cells seeded per well. C. Growth curves of 633 wild-type and $p 53^{-/}$cells cultured for 4 days in separate or co-culture conditions with a 634 starting cell number of $0.2 \times 10^{5}$ wild-type cells and $0.1 \times 10^{5} \mathrm{p} 53^{-/}$cells seeded per well. Data 635 were obtained from three independent experiments and are shown as the mean + SEM (A636 C). 


\section{REFERENCES}

638 Baker, N.E. (2020). Emerging mechanisms of cell competition. Nat Rev Genet 21, 683-697.

639 Bondar, T., and Medzhitov, R. (2010). p53-mediated hematopoietic stem and progenitor cell

640 competition. Cell stem cell 6, 309-322.

641 Bowling, S., Di Gregorio, A., Sancho, M., Pozzi, S., Aarts, M., Signore, M., M, D.S., Barbera,

642 J.P.M., Gil, J., and Rodriguez, T.A. (2018). P53 and mTOR signalling determine fitness

643 selection through cell competition during early mouse embryonic development. Nat

644 Commun 9, 1763.

645 Bowling, S., Lawlor, K., and Rodriguez, T.A. (2019). Cell competition: the winners and losers

646 of fitness selection. Development 146.

647 Claveria, C., Giovinazzo, G., Sierra, R., and Torres, M. (2013). Myc-driven endogenous cell

648 competition in the early mammalian embryo. Nature (London) 500, 39-44.

649 de la Cova, C., Abril, M., Bellosta, P., Gallant, P., and Johnston, L.A. (2004). Drosophila myc

650 regulates organ size by inducing cell competition. Cell 117, 107-116.

651 de la Cova, C., Senoo-Matsuda, N., Ziosi, M., Wu, C., Bellosta, P., Quinzii, C.M., and Johnston,

652 L.A. (2014). Supercompetitor Status of Drosophila Myc Cells Requires p53 as a Fitness

653 Sensor to Reprogram Metabolism and Promote Viability. Cell Metabolism 19, 1-14.

654 Dejosez, M., Ura, H., Brandt, V.L., and Zwaka, T.P. (2013). Safeguards for cell cooperation in

655 mouse embryogenesis shown by genome-wide cheater screen. Science 341, 1511-1514.

656 Diaz-Diaz, C., Fernandez de Manuel, L., Jimenez-Carretero, D., Montoya, M.C., Claveria, C.,

657 and Torres, M. (2017). Pluripotency Surveillance by Myc-Driven Competitive Elimination of

658 Differentiating Cells. Developmental cell 42, 585-599 e584.

659 Diaz-Diaz, C., and Torres, M. (2019). Insights into the quantitative and dynamic aspects of

660 Cell Competition. Curr Opin Cell Biol 60, 68-74.

661 Fuchs, Y., and Steller, H. (2015). Live to die another way: modes of programmed cell death

662 and the signals emanating from dying cells. Nature reviews 16, 329-344.

663 Germani, F., Hain, D., Sternlicht, D., Moreno, E., and Basler, K. (2018). The Toll pathway

664 inhibits tissue growth and regulates cell fitness in an infection-dependent manner. Elife 7.

665 Gradeci, D., Bove, A., Vallardi, G., Lowe, A.R., Banerjee, S., and Charras, G. (2021). Cell-scale

666 biophysical determinants of cell competition in epithelia. Elife 10.

667 Hashimoto, M., and Sasaki, H. (2019). Epiblast Formation by TEAD-YAP-Dependent

668 Expression of Pluripotency Factors and Competitive Elimination of Unspecified Cells.

669 Developmental cell 50, 139-154 e135.

670 Johnston, L.A. (2014). Socializing with MYC: cell competition in development and as a model

671 for premalignant cancer. Cold Spring Harb Perspect Med 4, a014274.

672 Katsukawa, M., Ohsawa, S., Zhang, L., Yan, Y., and Igaki, T. (2018). Serpin Facilitates Tumor-

673 Suppressive Cell Competition by Blocking Toll-Mediated Yki Activation in Drosophila. Curr

674 Biol 28, 1756-1767 e1756.

675 Kursawe, J., Baker, R.E., and Fletcher, A.G. (2018). Approximate Bayesian computation

676 reveals the importance of repeated measurements for parameterising cell-based models of

677 growing tissues. J Theor Biol 443, 66-81.

678 Lawlor, K., Marques-Torrejon, M.A., Dharmalingham, G., El-Azhar, Y., Schneider, M.D.,

679 Pollard, S.M., and Rodriguez, T.A. (2020). Glioblastoma stem cells induce quiescence in

680 surrounding neural stem cells via Notch signaing. Genes Dev. 
681 Lee, S.W., and Morishita, Y. (2017). Possible roles of mechanical cell elimination intrinsic to

682 growing tissues from the perspective of tissue growth efficiency and homeostasis. PLoS

683 Comput Biol 13, e1005651.

684 Levayer, R., Hauert, B., and Moreno, E. (2015). Cell mixing induced by myc is required for

685 competitive tissue invasion and destruction. Nature (London) 524, 476-480.

686 Lima, A., Lubatti, G., Burgstaller, J., Hu, D., Green, A.P., Di Gregorio, A., Zawadzki, T.,

687 Pernaute, B., Mahammadov, E., Perez-Montero, S., et al. (2021). Cell competition acts as a

688 purifying selection to eliminate cells with mitochondrial defects during early mouse

689 development. Nature Metabolism.

690 Madan, E., Gogna, R., and Moreno, E. (2018). Cell competition in development: information

691 from flies and vertebrates. Curr Opin Cell Biol 55, 150-157.

692 Madan, E., Pelham, C.J., Nagane, M., Parker, T.M., Canas-Marques, R., Fazio, K., Shaik, K.,

693 Yuan, Y., Henriques, V., Galzerano, A., et al. (2019). Flower isoforms promote competitive

694 growth in cancer. Nature (London) 572, 260-264.

695 Maruyama, T., and Fujita, Y. (2017). Cell competition in mammals - novel homeostatic

696 machinery for embryonic development and cancer prevention. Curr Opin Cell Biol 48, 106-

697112.

698 Merkle, F.T., Ghosh, S., Kamitaki, N., Mitchell, J., Avior, Y., Mello, C., Kashin, S., Mekhoubad,

699 S., Ilic, D., Charlton, M., et al. (2017). Human pluripotent stem cells recurrently acquire and

700 expand dominant negative P53 mutations. Nature (London).

701 Meyer, S.N., Amoyel, M., Bergantinos, C., de la Cova, C., Schertel, C., Basler, K., and

702 Johnston, L.A. (2014). An ancient defense system eliminates unfit cells from developing

703 tissues during cell competition. Science 346, 1258236.

704 Moreno, E., and Basler, K. (2004). dMyc transforms cells into super-competitors. Cell 117,

705 117-129.

706 Nishikawa, S., Takamatsu, A., Ohsawa, S., and Igaki, T. (2016). Mathematical model for cell

707 competition: Predator-prey interactions at the interface between two groups of cells in

708 monolayer tissue. J Theor Biol 404, 40-50.

709 Pilley, S., Rodriguez, T.A., and Vousden, K.H. (2021). Mutant p53 in cell-cell interactions.

710 Genes Dev 35, 433-448.

711 Price, C.J., Stavish, D., Gokhale, P.J., Stevenson, B.A., Sargeant, S., Lacey, J., Rodriguez, T.A.,

712 and Barbaric, I. (2021). Genetically variant human pluripotent stem cells selectively

713 eliminate wild-type counterparts through YAP-mediated cell competition. Developmental

714 cell.

715 Ram, Y., Dellus-Gur, E., Bibi, M., Karkare, K., Obolski, U., Feldman, M.W., Cooper, T.F.,

716 Berman, J., and Hadany, L. (2019). Predicting microbial growth in a mixed culture from

717 growth curve data. Proc Natl Acad Sci U S A 116, 14698-14707.

718 Rhiner, C., Lopez-Gay, J.M., Soldini, D., Casas-Tinto, S., Martin, F.A., Lombardia, L., and

719 Moreno, E. (2010). Flower forms an extracellular code that reveals the fitness of a cell to its

720 neighbors in Drosophila. Developmental cell 18, 985-998.

721 Sancho, M., Di-Gregorio, A., George, N., Pozzi, S., Sanchez, J.M., Pernaute, B., and Rodriguez,

722 T.A. (2013). Competitive interactions eliminate unfit embryonic stem cells at the onset of

723 differentiation. Developmental cell 26, 19-30.

724 Schindelin, J., Arganda-Carreras, I., Frise, E., Kaynig, V., Longair, M., Pietzsch, T., Preibisch, S.,

725 Rueden, C., Saalfeld, S., Schmid, B., et al. (2012). Fiji: an open-source platform for biological-

726 image analysis. Nature methods 9, 676-682. 
727 Shockley, E.M., Vrugt, J.A., and Lopez, C.F. (2018). PyDREAM: high-dimensional parameter

728 inference for biological models in python. Bioinformatics 34, 695-697.

729 Shraiman, B.I. (2005). Mechanical feedback as a possible regulator of tissue growth. Proc

$730 \quad$ Natl Acad Sci U S A 102, 3318-3323.

731 Tait, S.W., and Green, D.R. (2013). Mitochondrial regulation of cell death. Cold Spring Harb

732 Perspect Biol 5.

733 Toni, T., Welch, D., Strelkowa, N., Ipsen, A., and Stumpf, M.P. (2009). Approximate Bayesian

734 computation scheme for parameter inference and model selection in dynamical systems. J R

735 Soc Interface 6, 187-202.

736 Tsuboi, A., Ohsawa, S., Umetsu, D., Sando, Y., Kuranaga, E., Igaki, T., and Fujimoto, K. (2018).

737 Competition for Space Is Controlled by Apoptosis-Induced Change of Local Epithelial

738 Topology. Curr Biol 28, 2115-2128 e2115.

739 Villa Del Campo, C., Claveria, C., Sierra, R., and Torres, M. (2014). Cell competition promotes

740 phenotypically silent cardiomyocyte replacement in the Mammalian heart. Cell Rep 8, 1741-

7411751.

742 Vishwakarma, M., and Piddini, E. (2020). Outcompeting cancer. Nat Rev Cancer 20, 187-198.

743 Wagstaff, L., Goschorska, M., Kozyrska, K., Duclos, G., Kucinski, I., Chessel, A., Hampton-

744 O'Neil, L., Bradshaw, C.R., Allen, G.E., Rawlins, E.L., et al. (2016). Mechanical cell

745 competition kills cells via induction of lethal p53 levels. Nat Commun 7, 11373.

746 Zhang, G., Xie, Y., Zhou, Y., Xiang, C., Chen, L., Zhang, C., Hou, X., Chen, J., Zong, H., and Liu,

747 G. (2017). p53 pathway is involved in cell competition during mouse embryogenesis. Proc

748 Natl Acad Sci U S A 114, 498-503.

749 Zheng, C., Hu, Y., Sakurai, M., Pinzon-Arteaga, C.A., Li, J., Wei, Y., Okamura, D., Ravaux, B.,

750 Barlow, H.R., Yu, L., et al. (2021). Cell competition constitutes a barrier for interspecies

751 chimerism. Nature (London) 592, 272-276.

752 
FIGURE 1

Cell competition assays - Pluripotency

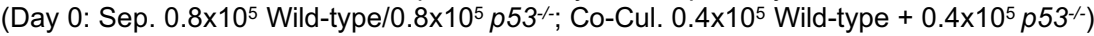

A

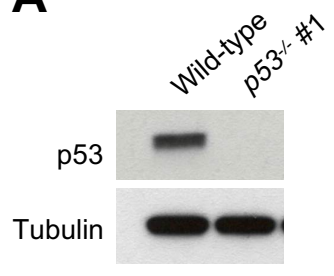

SEPARATE CULTURE

B $p 53^{--} \quad$ Wild-type

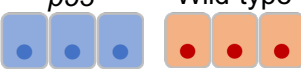

\section{CO-CULTURE}
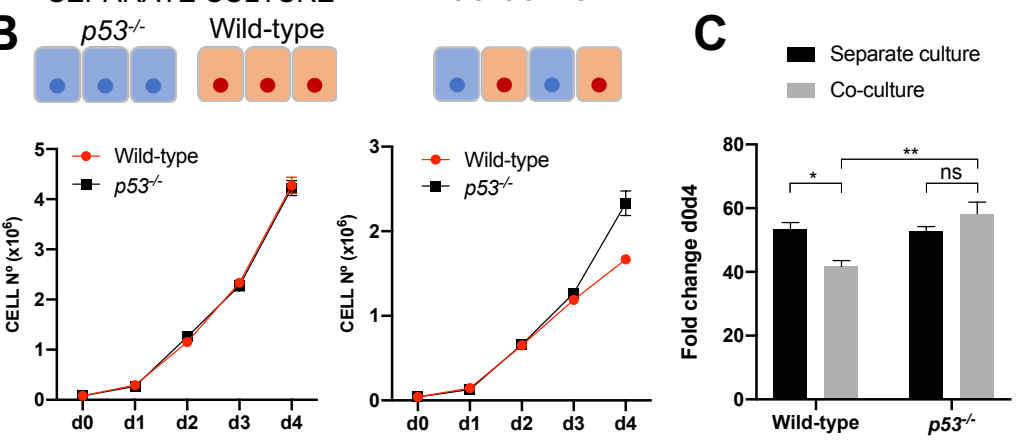

D

Cell competition assays - Differentiation

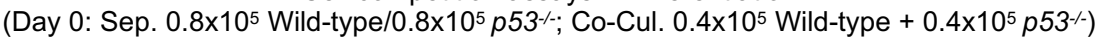
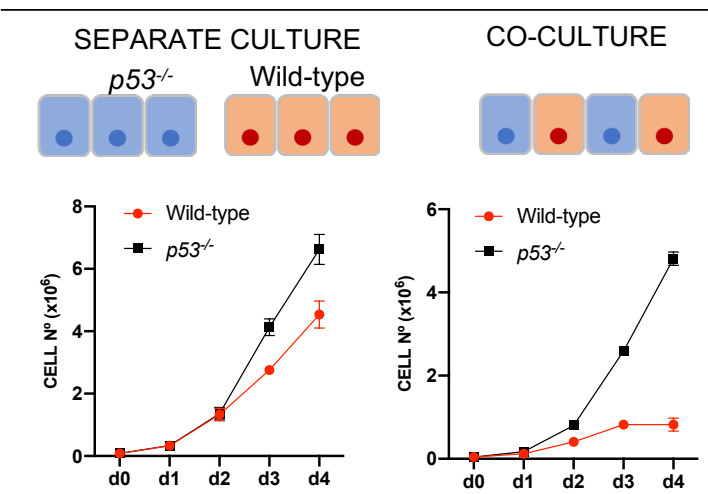

$\mathbf{F}$

$$
\begin{aligned}
& \text { - Wild-type Pluripotency YIII/ Wild-type Differentiation } \\
& p 53^{-/} \text {Pluripotency } p 53^{- \text {- Differentiation }}
\end{aligned}
$$
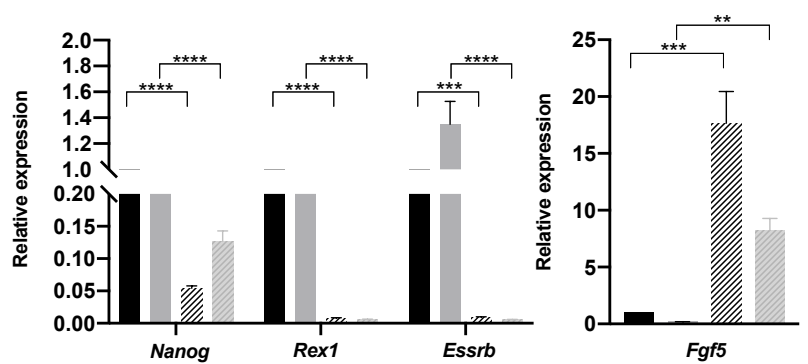

$\mathbf{H}$

$$
\begin{aligned}
& \text { - Separate culture } \\
& \text { Co-culture }
\end{aligned}
$$

Day 3

Day 4
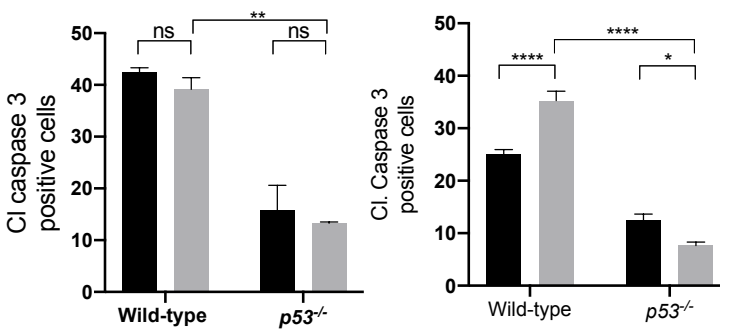
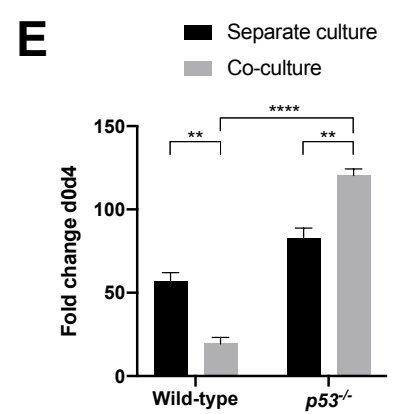

G

Separate culture Co-culture

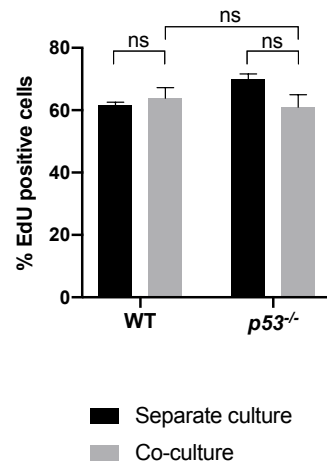

Day 3

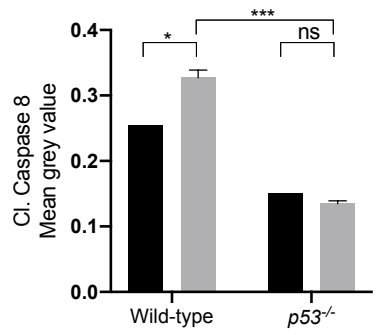


FIGURE 2

A

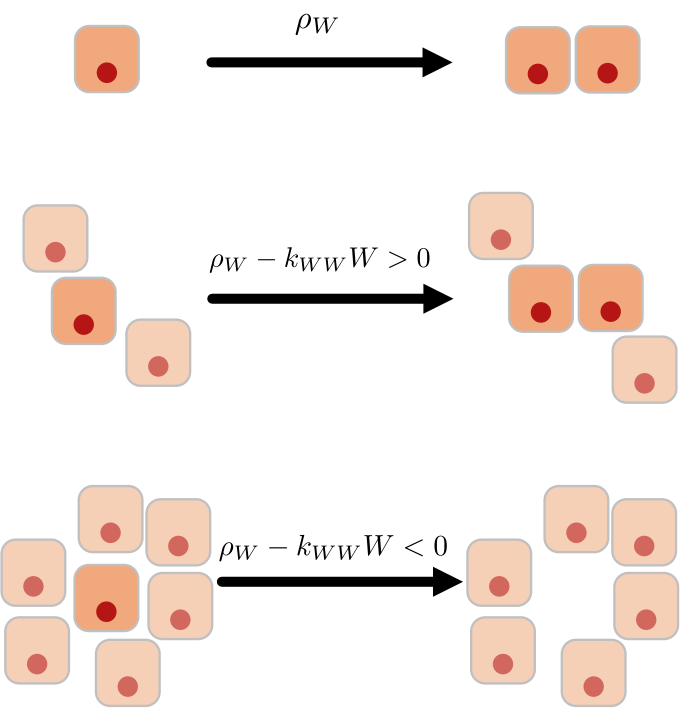

C
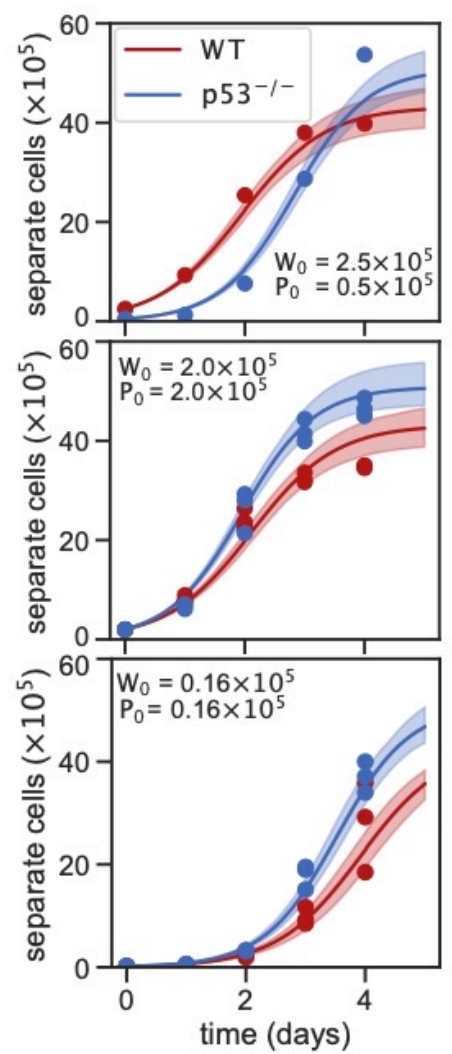
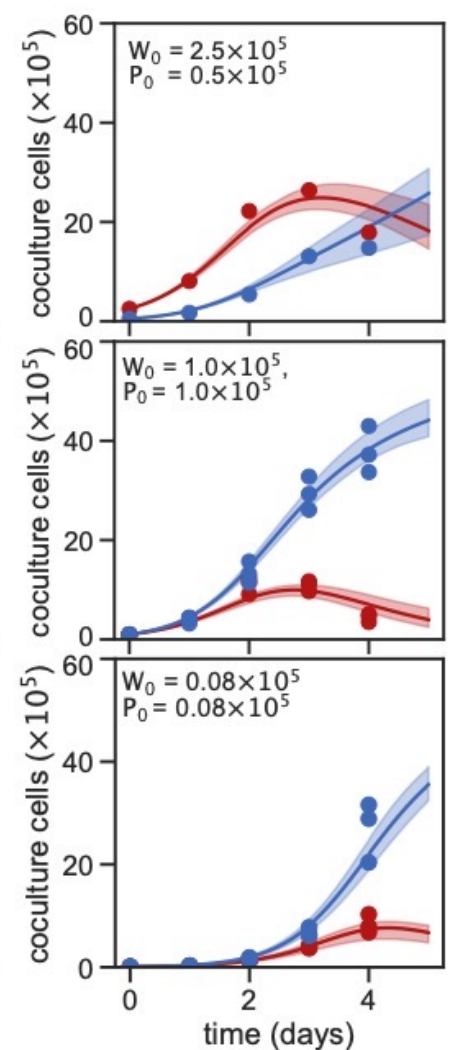

B

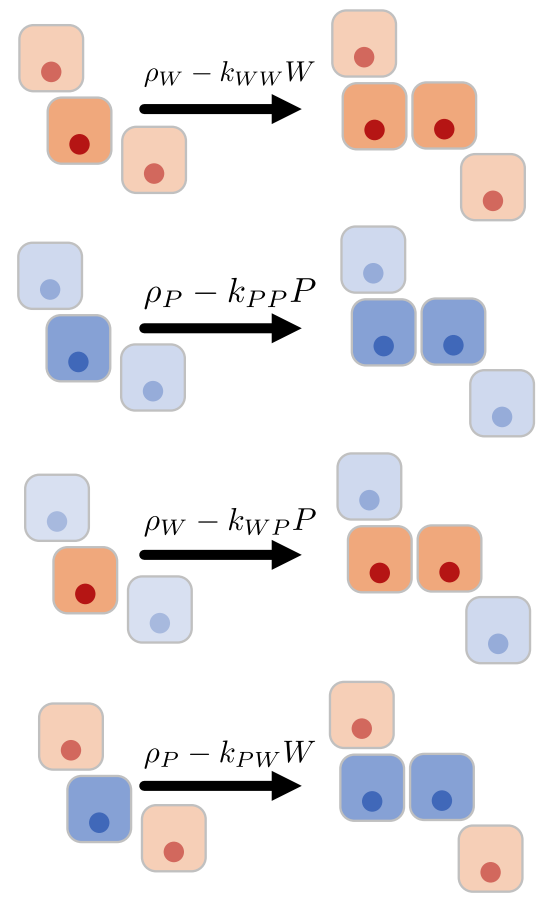

D
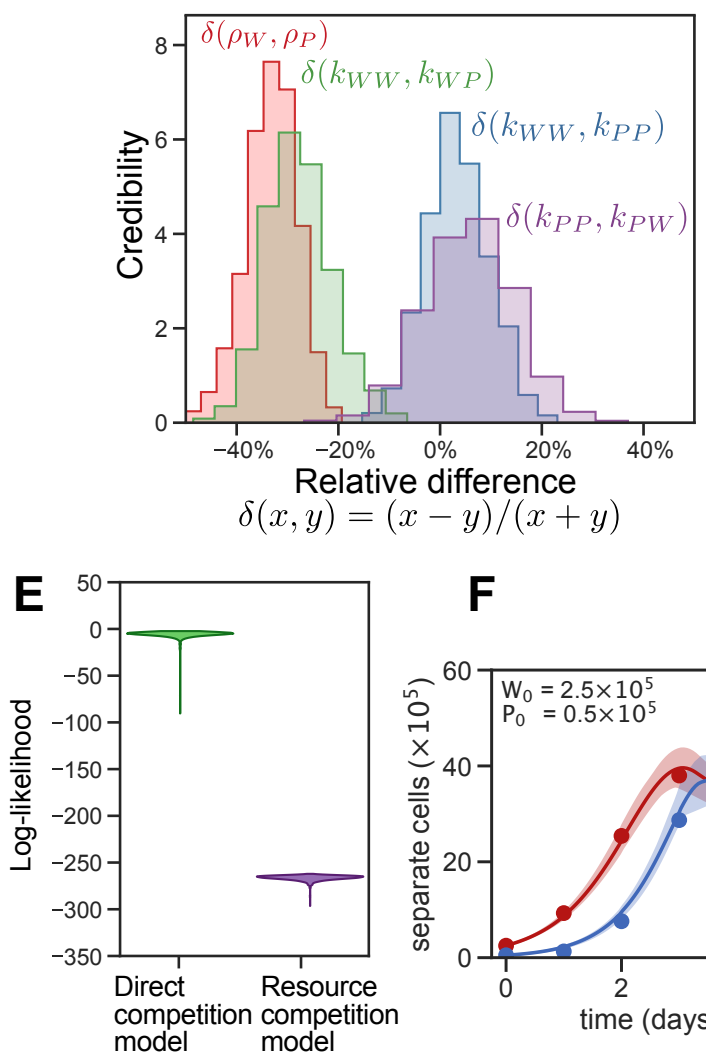

F

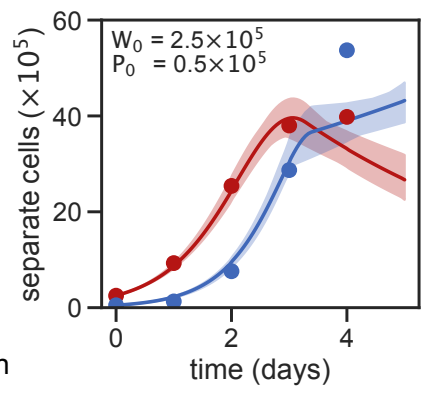


FIGURE 3

A

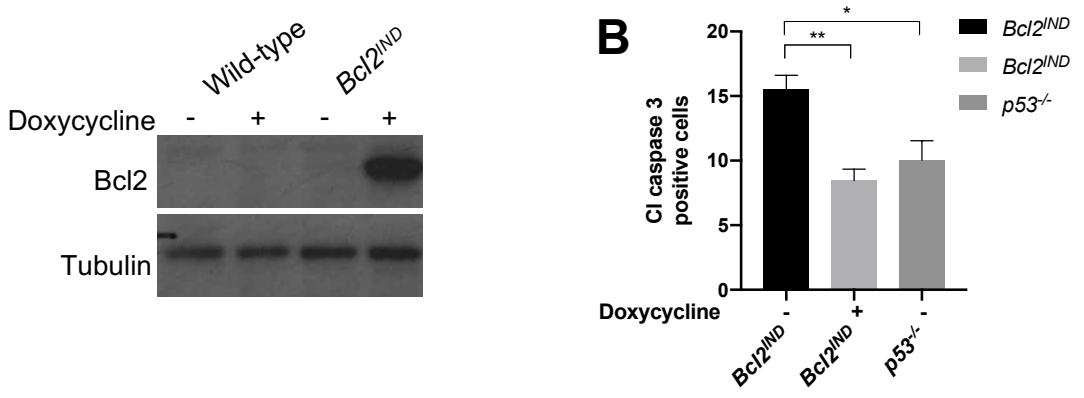

Cell competition assays - Differentiation (- Doxycycline)

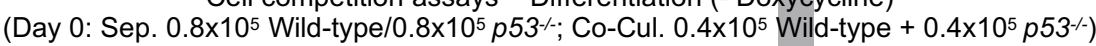

D

\section{SEPARATE CULTURE}

$B C / 2^{I N D}$

$p 53^{--G F P}$
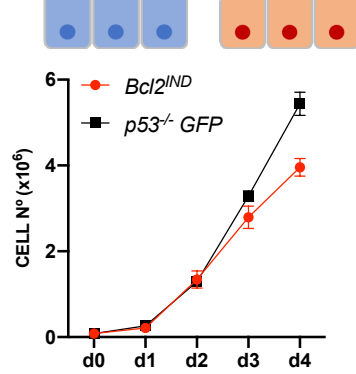

Cell competition assays - Differentiation (+ Doxycycline)

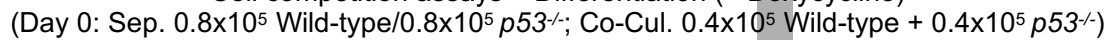

$\mathbf{F}$
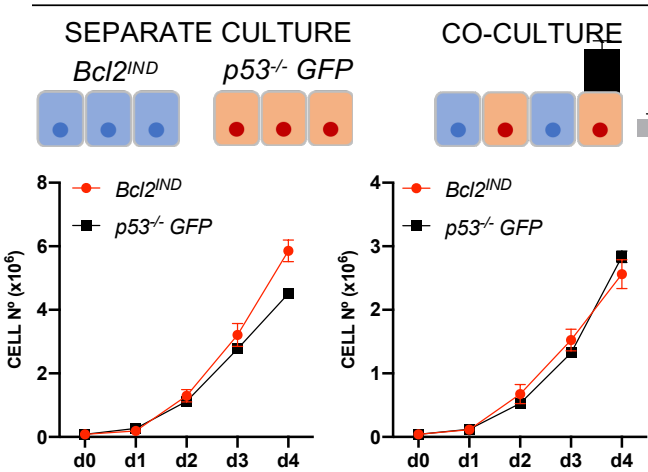

E - Separate culture - Co-culture
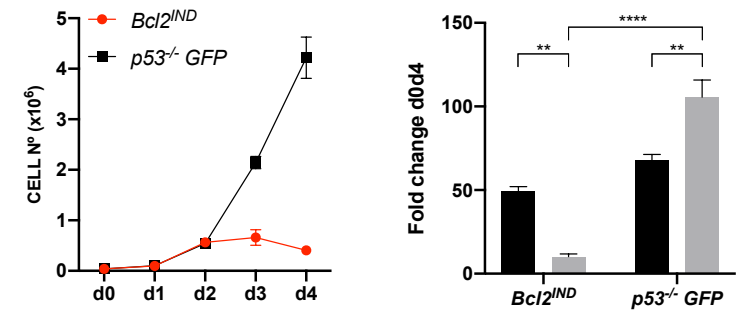

H
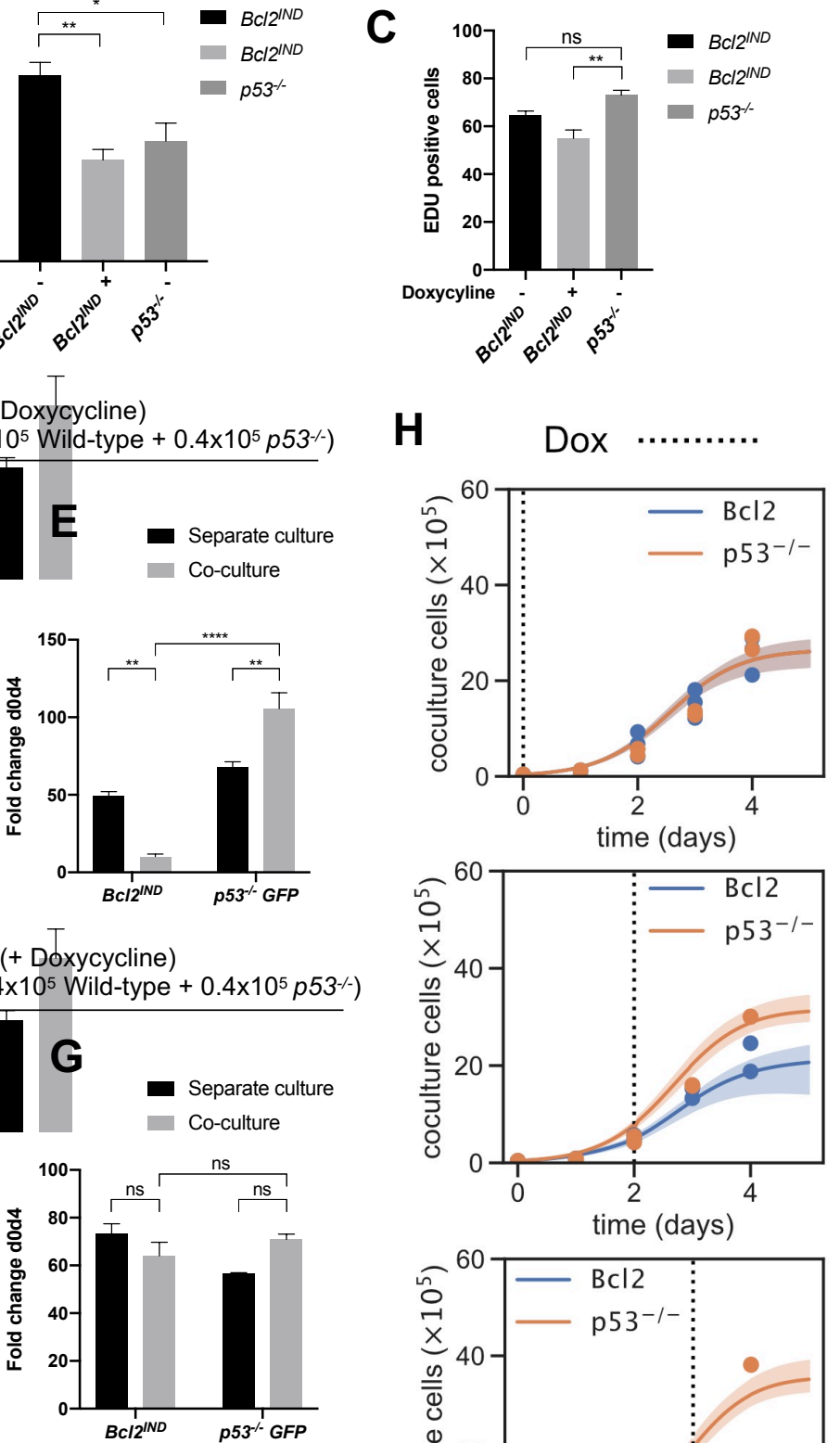

I
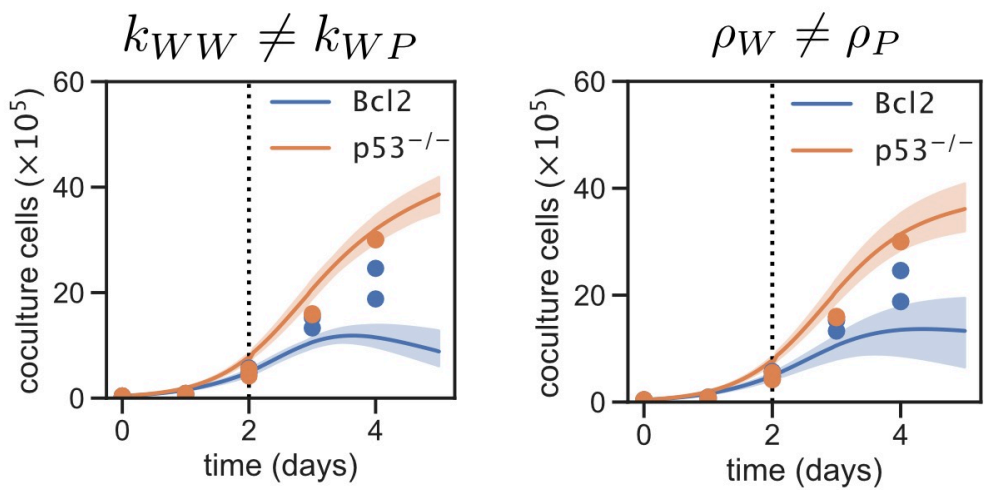
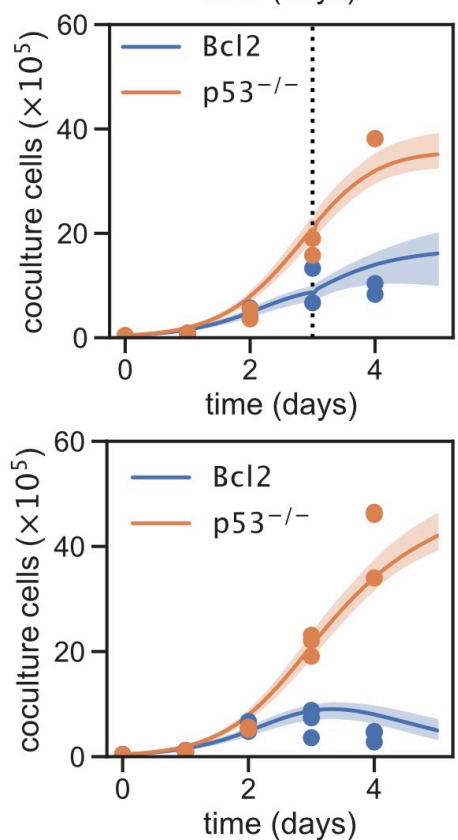
A

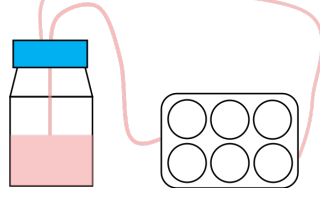

SEPARATE CULTURE

p53\% Wild-type

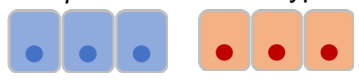

- control

- perfusion

CO-CULTURE

B
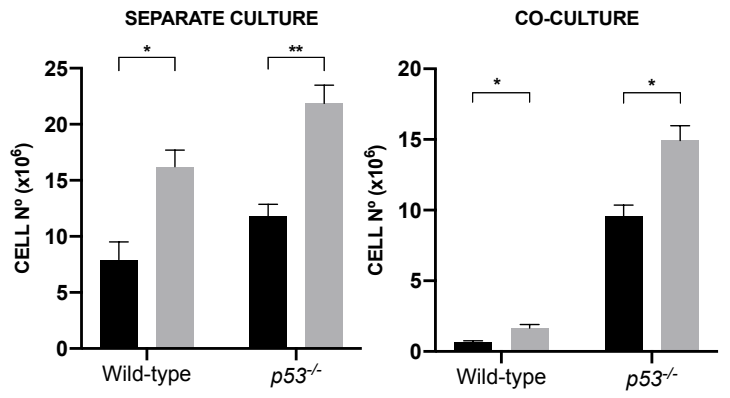

Wild-type $\quad p 53^{-/}$

D
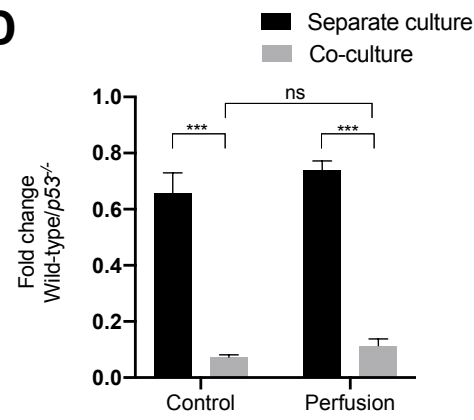

CO-CULTURE
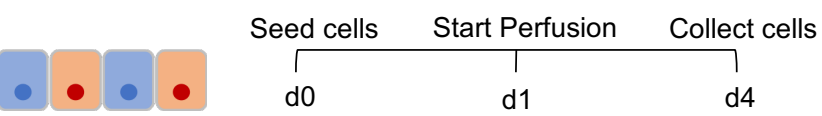

d0

d1

d4
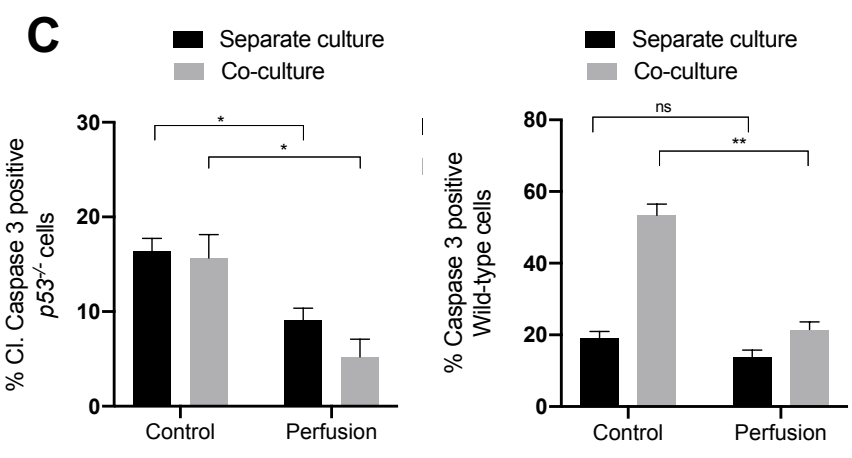

Conditioned media assays - Differentiation

E

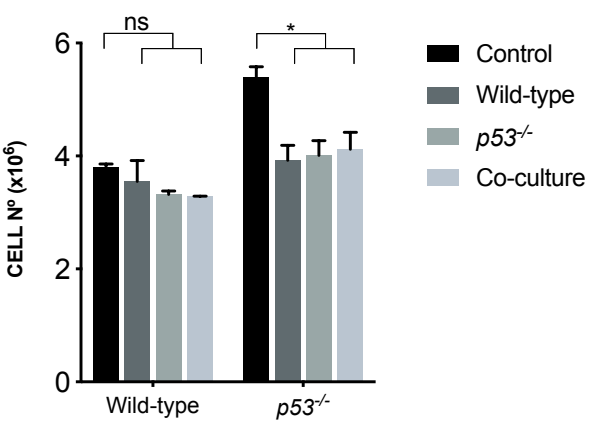

Cell competition fences assays - Differentiation

$\mathbf{F}$ $p 53^{--}$

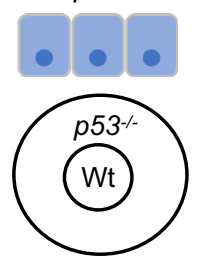

Seed cells

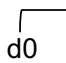

H

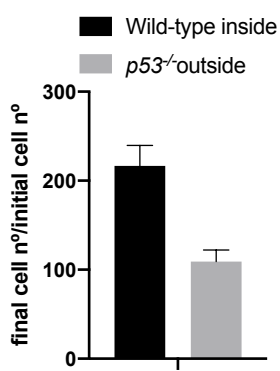

Wild-type

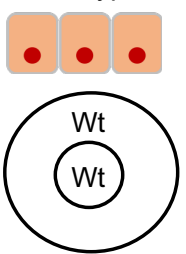

Remove fences Collect cells
G

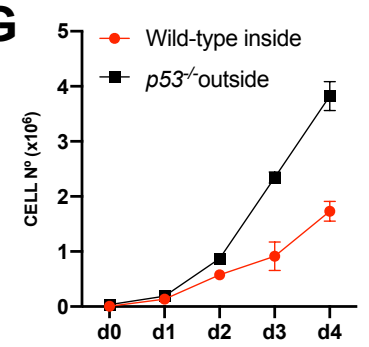

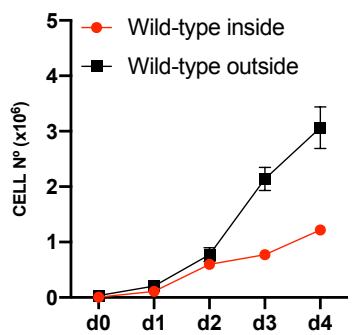

d4
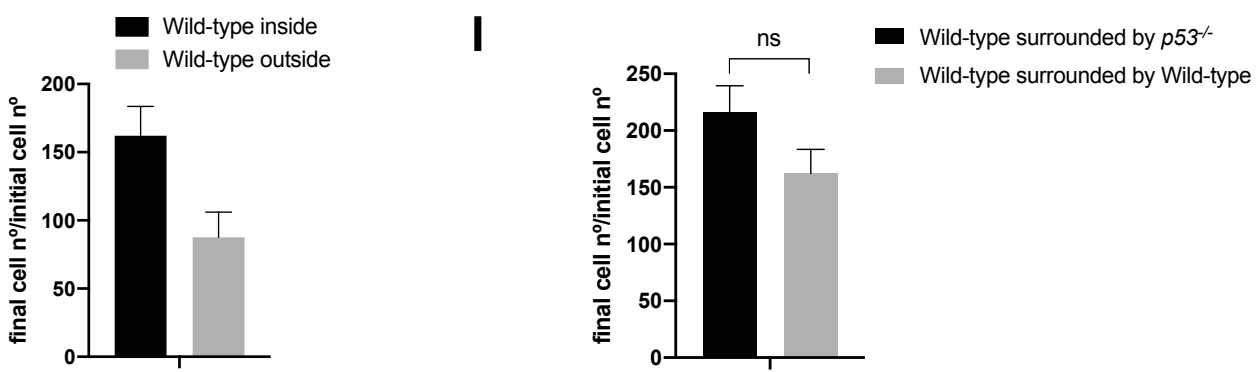
FIGURE 5

Co-culture (Day 0: $0.4 \times 10^{5}$ Wild-type $+0.4 \times 10^{5} p 53^{-/-}$)

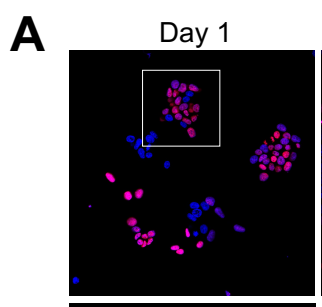

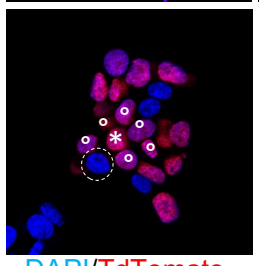

DAPI/TdTomato
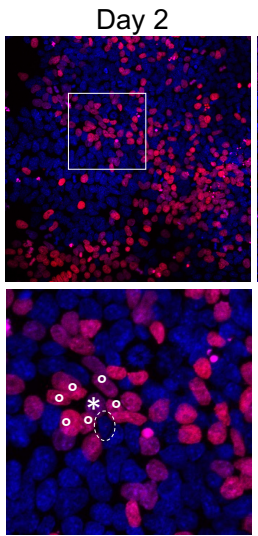

DAPI/TdTomato
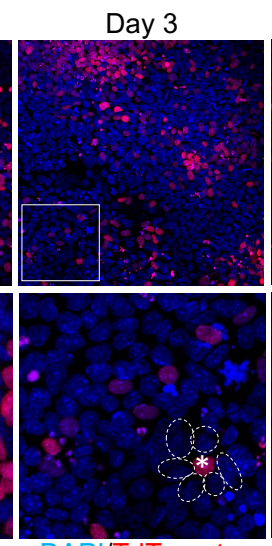

DAPI/TdTomato
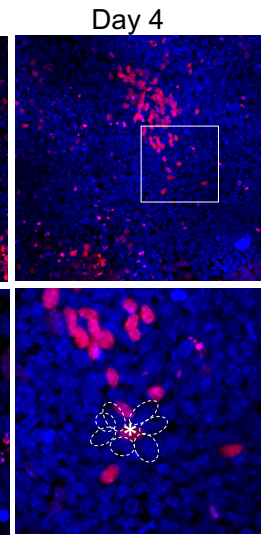

DAPI/TdTomato
B
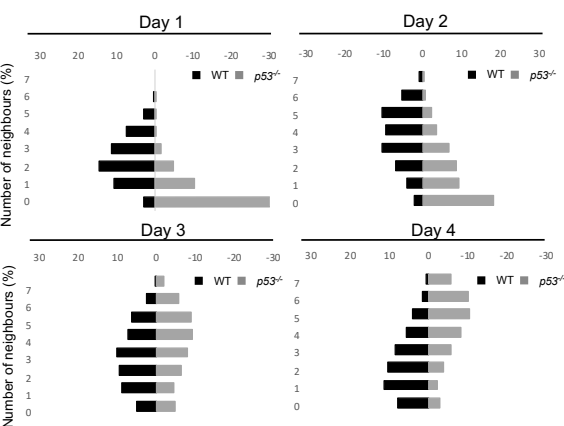

Co-culture (Day 0: $0.4 \times 10^{5} \mathrm{Bc/2} / \mathrm{IND}+0.4 \times 10^{5} \mathrm{p} 53^{-/-}$)

C

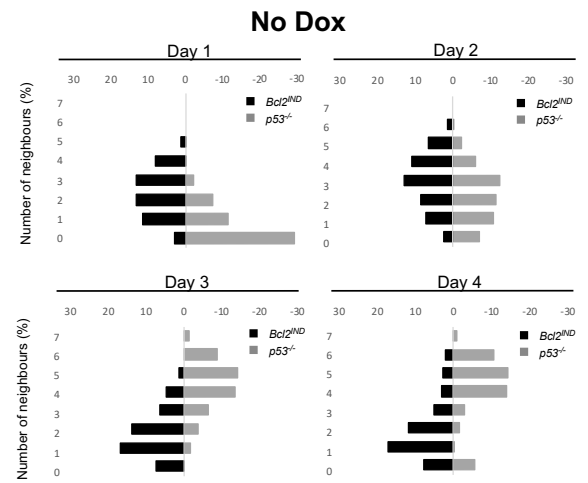

D

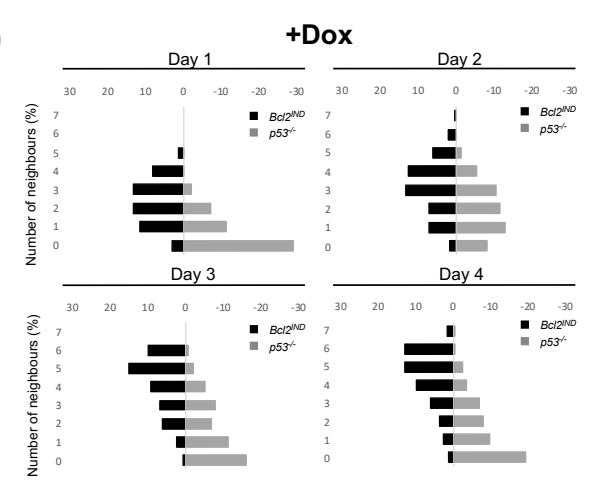

G

Co-culture

(Day 0: $5 \times 10^{5}$ Wild-type $+5 \times 10^{5} p 53^{-/-}$)

(Day 0: $0.08 \times 10^{5}$ Wild-type $+0.08 \times 10^{5} p 53^{-/-}$)

F

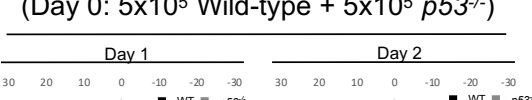

(Day 0: $2 \times 10^{5}$ Wild-type $+1 \times 10^{5} p 53^{-/-}$)

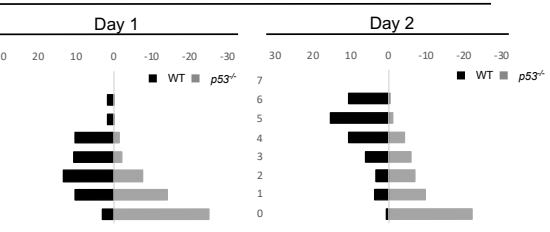

Day 4

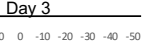

$10 \quad 0 \quad-10-20-30-40-50$
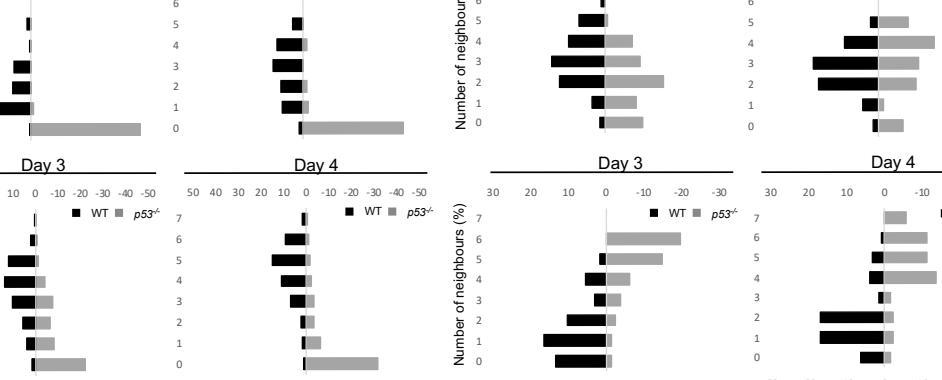

Day 4

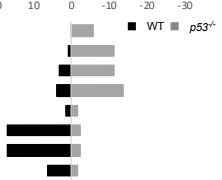

Day 3

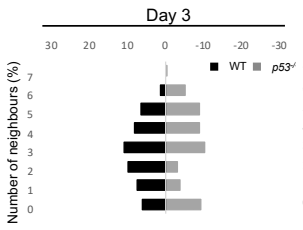

Day 4

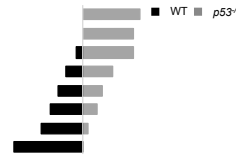


A

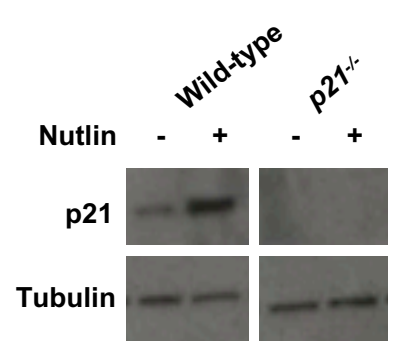

B

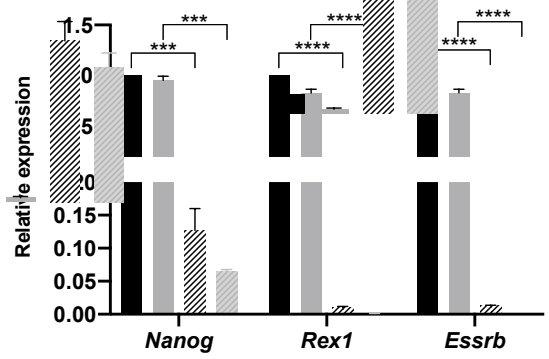
WII/. Wild-type Differentiation p21 $21^{-/}$Differentiation

Cell competition assays - Differentiation

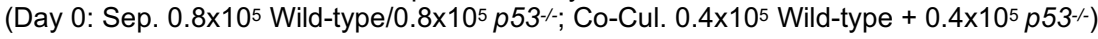
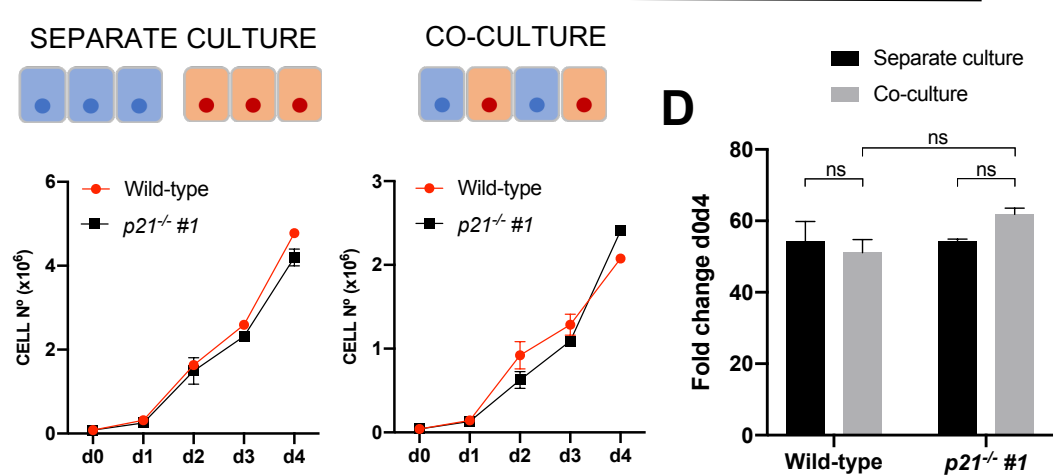

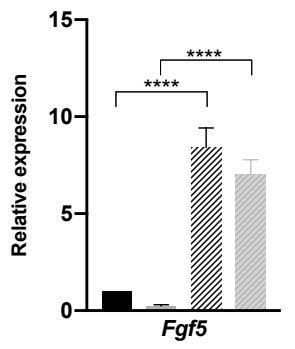

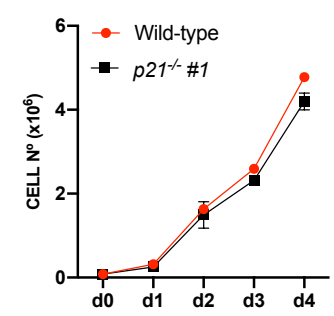

C

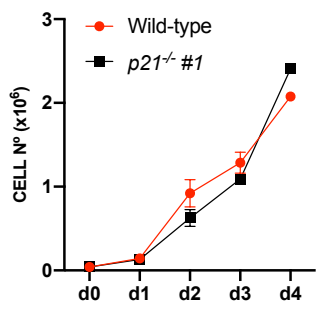

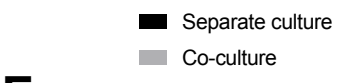

$\mathbf{E}$

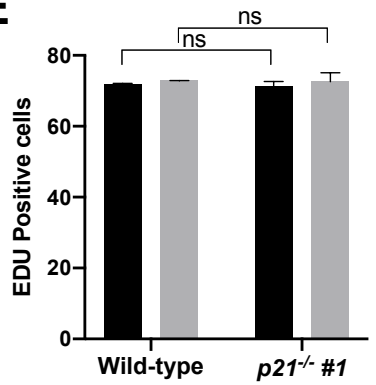


A

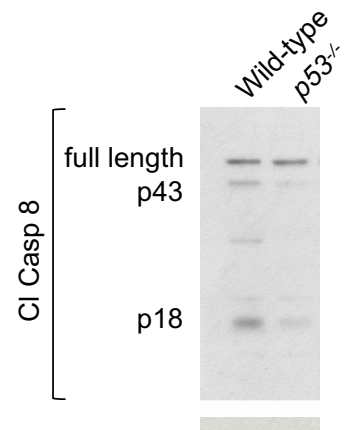

Tubulin
B

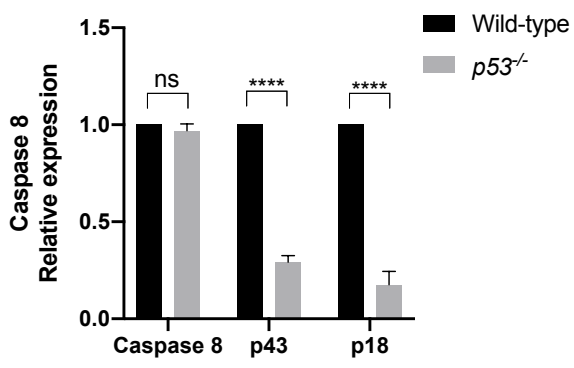

Day 3 Differentiation

C
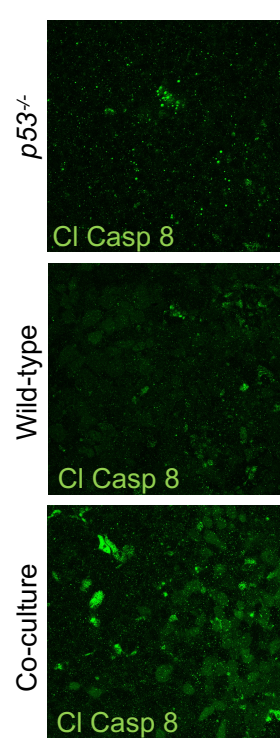
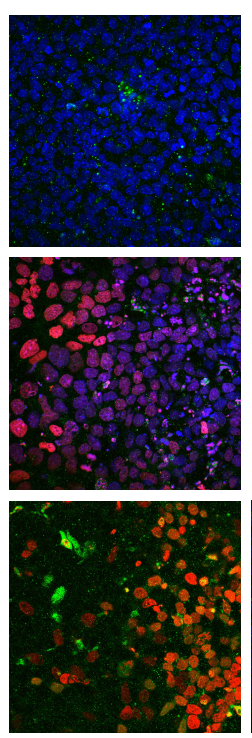

D

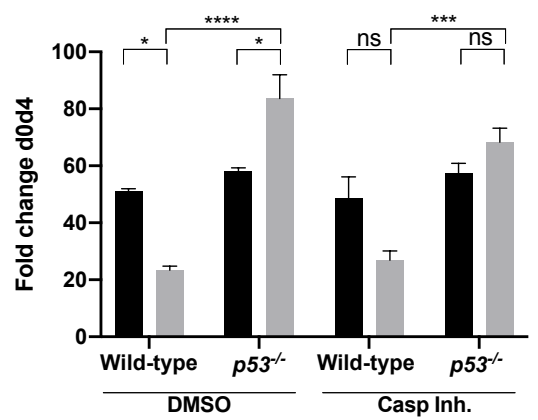




\section{FIGURE S3}

A
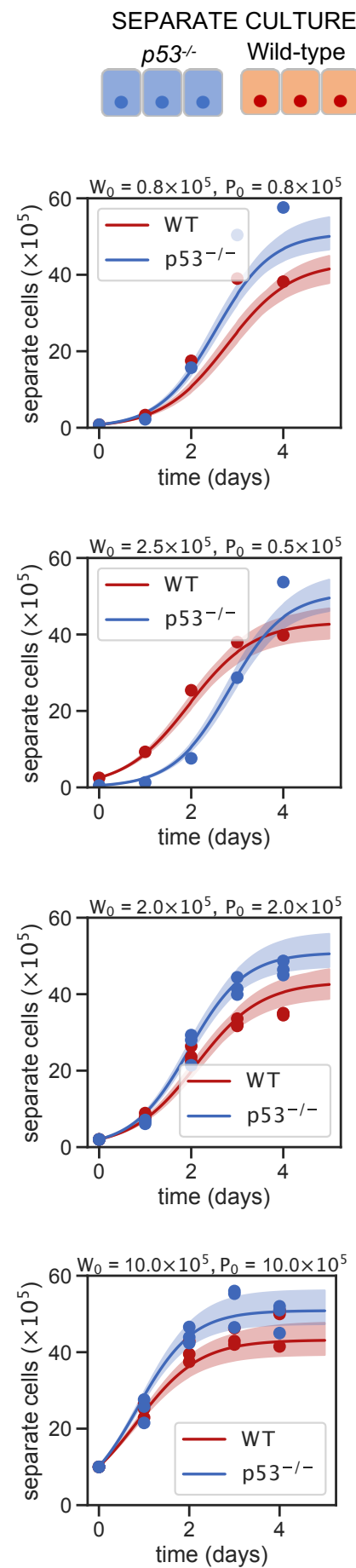

CO-CULTURE
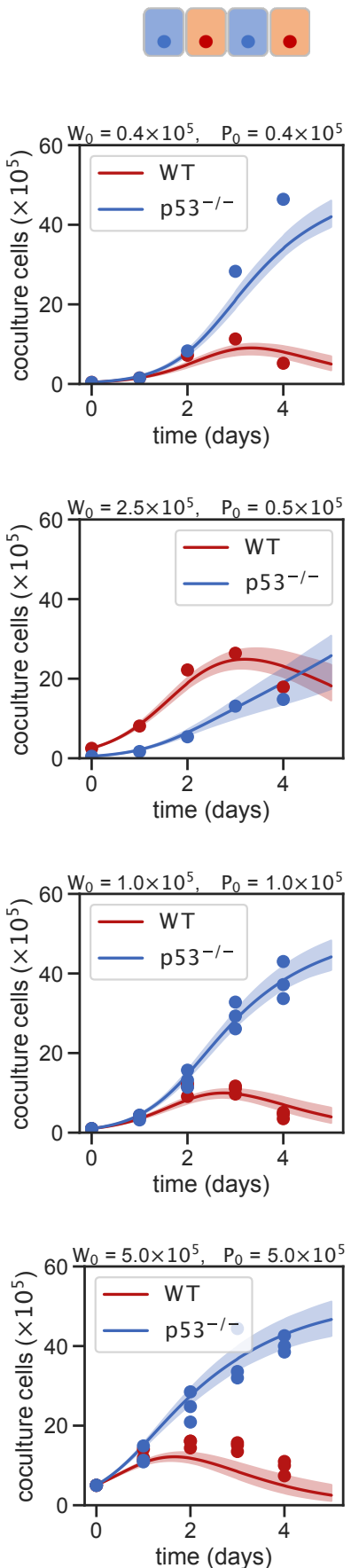

SEPARATE CULTURE

CO-CULTURE p53\%

Wild-type
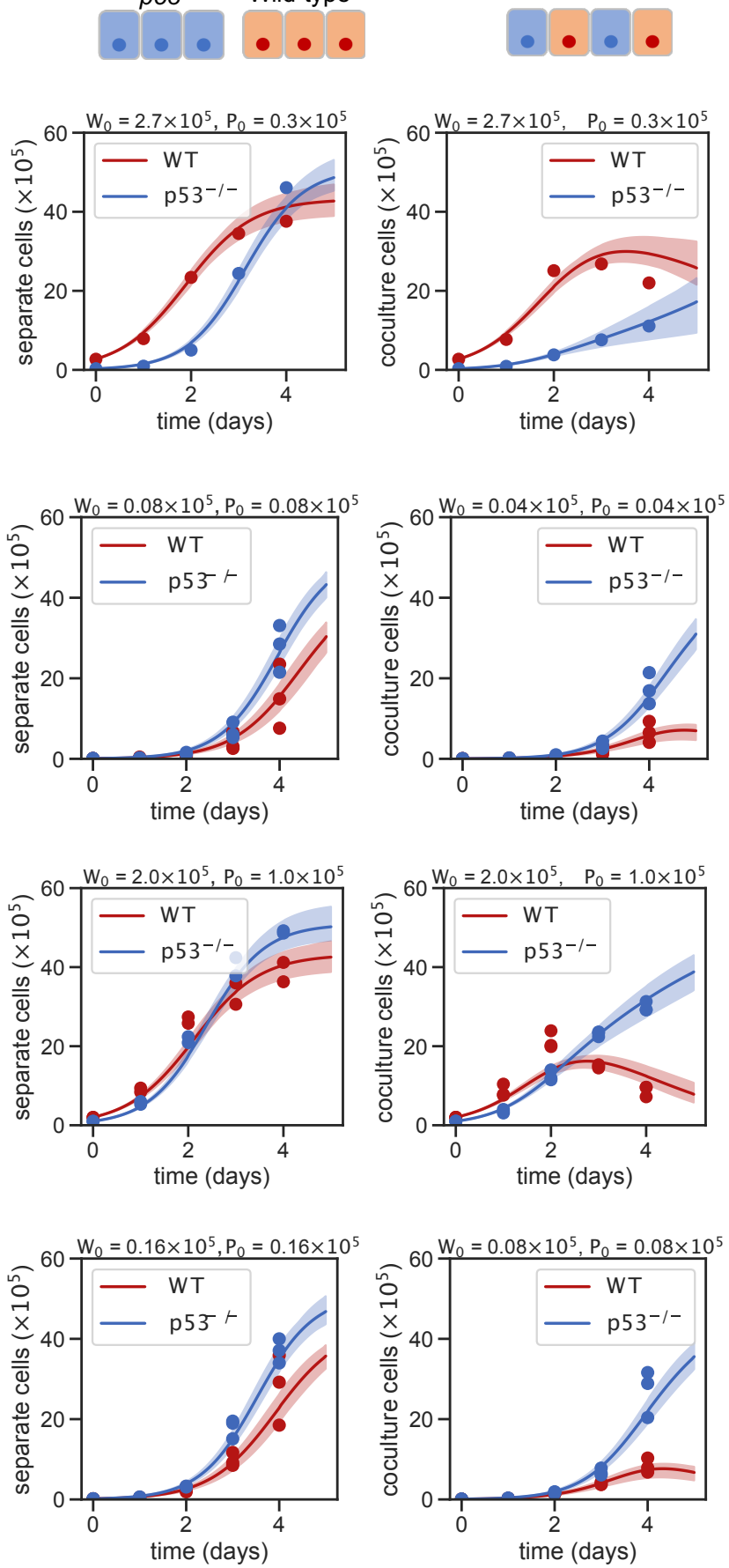
FIGURE S4
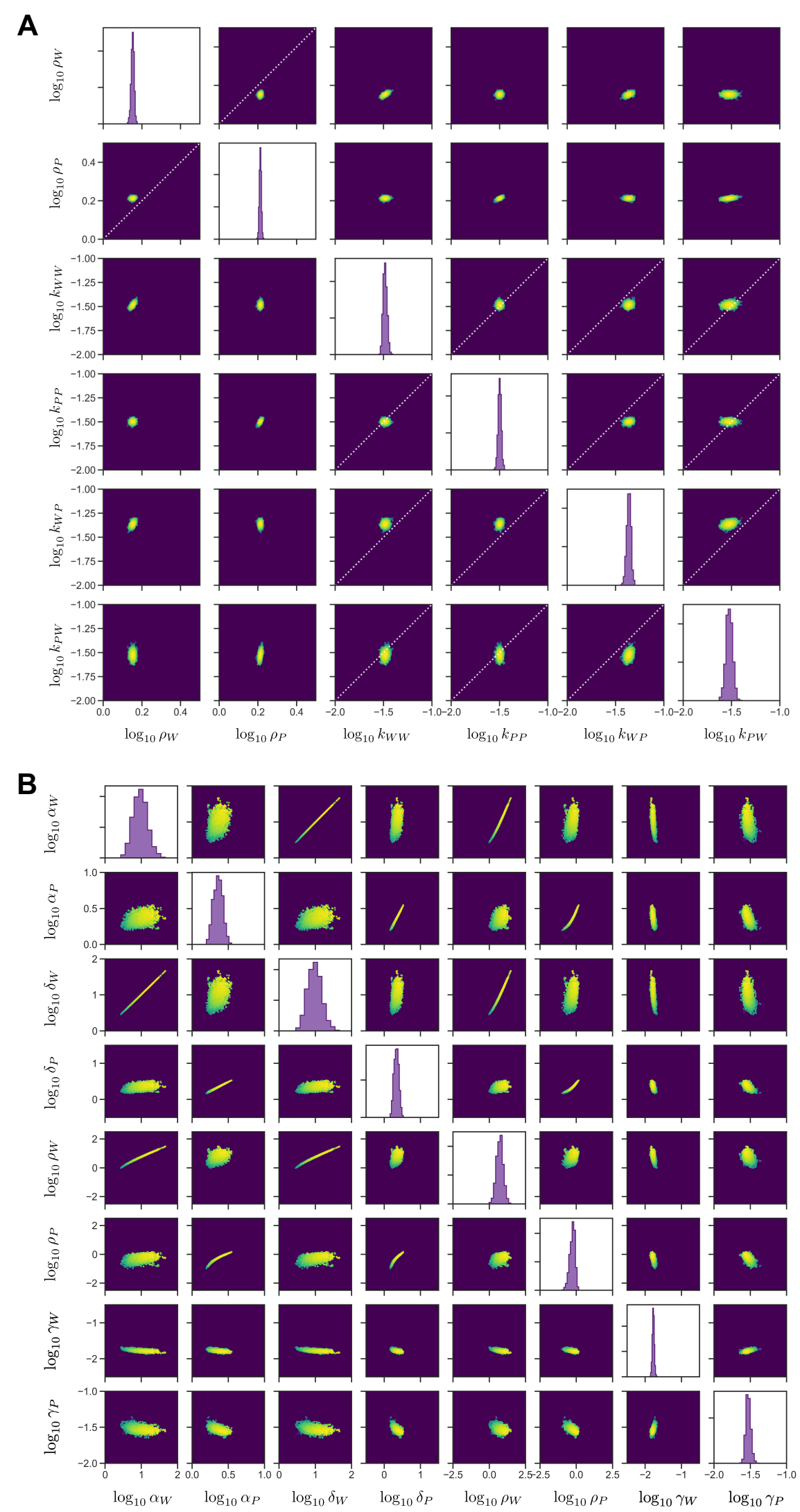


\section{FIGURE S5}

A

Cell competition assays

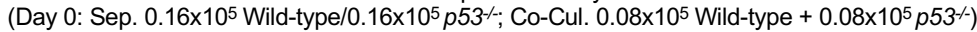

B

$\begin{array}{ccc}\text { SEPARATE CUTURE } & \text { CO-CUTURE } \\ \text { p53\% } & \text { Wild-type }\end{array}$
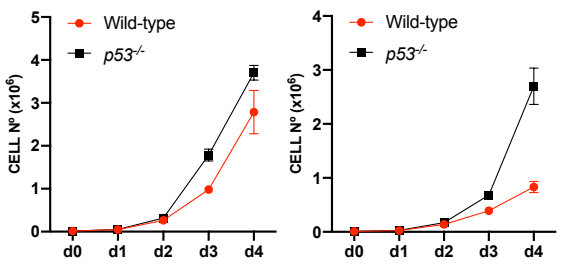

Cell competition assays

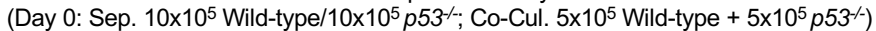
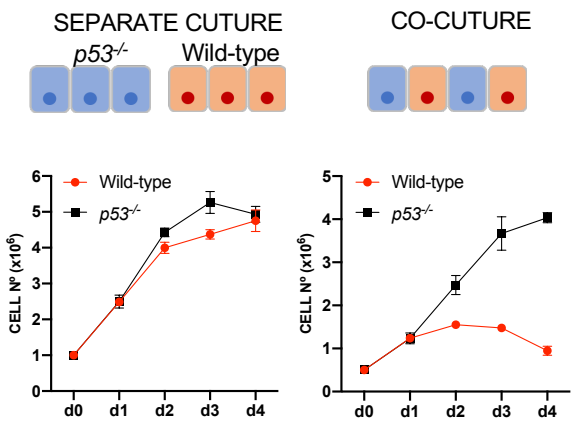

C

Cell competition assays

(Day 0: Sep. $2 \times 10^{5}$ Wild-type/ $1 \times 10^{5} p 53^{--;}$; Co-Cul. $2 \times 10^{5}$ Wild-type + $1 \times 10^{5} p 53^{--}$)
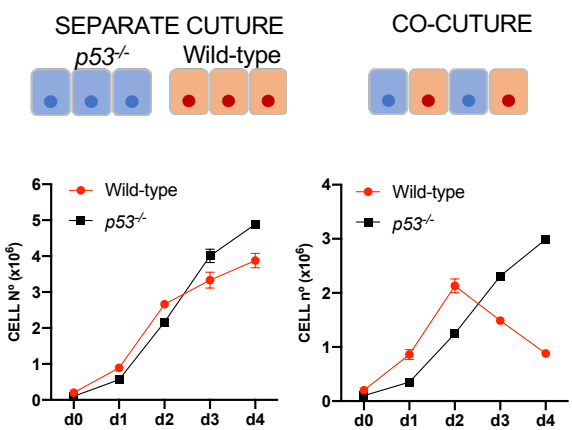